\title{
Acupuncture methods for allergic rhinitis: a systematic review and bayesian meta-analysis of randomized controlled trials
}

\author{
Zihan Yin, Guoyan Geng, Guixing Xu, Ling Zhao ${ }^{*}$ and Fanrong Liang ${ }^{*}$ (i)
}

\begin{abstract}
Background: Allergic rhinitis (AR) is a common symptomatic, inflammatory, and immunological disorder of nasal mucosa. Multiple clinical trials and systematic reviews have implicated acupuncture methods as potentially effective treatment strategies for AR, however, considering the great burden of $A R$, it is crucial to explore the most recent clinical evidence supporting acupuncture in AR. Besides, the methodologies reported in previous studies as well as those commonly applied during clinical practices greatly vary. Herein, we conducted network meta-analysis to compare the effectiveness of diverse acupuncture methods for AR treatment.

Methods: We conducted a literature search for relevant reports published from inception to 1 July 2020 in several scientific databases, including PubMed, Embase, Cochrane library, Web of Science, CNKI, WF, VIP, CBM, AMED as well as related registration platforms. Primary outcomes as reported in the identified studies were assessed using nasal symptoms. All Meta-analyses were performed with RevMan, ADDIS, and STATA software. To ensure consistency among our reviewers, the intra-class correlation coefficient was used.
\end{abstract}

Results: Exactly 39 studies with 3433 participants were covered in this meta-analysis. The meta-analysis demonstrated that all acupuncture types were superior to sham acupuncture in terms of total nasal symptom score and rhinoconjunctivitis quality of life questionnaire. Moxibustion was recommended as the most effective intervention as it reduced nasal symptoms in 6 treatments. On the other hand, manual acupuncture plus conventional medicine was recommended as the most effective intervention in improving the quality of life in 9 treatments. Notably, moxibustion was recommended as the most effective intervention that changed the content of IgE in 9 treatments. Moreover, adverse events of these interventions were acceptable.

Conclusion: Our findings revealed that all acupuncture methods are effective and safe for AR. Moreover, either moxibustion or manual acupuncture plus conventional medicine are potentially the most effective treatment strategies for AR. Based on these findings, it is evident that acupuncture therapy is not inferior to pharmacologic therapy. Therefore, for AR patients who are either unresponsive to conventional medicine or are intolerant to adverse events, acupuncture therapy should be administered. However, the quality of these included trials was mainly ranked as moderate quality, we recommend additional well-designed RCTs with larger sample sizes to validate these findings.

Keywords: Acupuncture, Allergic rhinitis, Systematic review, Network meta-analysis

*Correspondence: zhaoling@cdutcm.edu.cn; acuresearch@126.com School of Acu-Mox and Tuina, Chengdu University of Traditional Chinese Medicine, 37 Shi Er Qiao Road, Chengdu 610075, China

\section{Background}

Allergic rhinitis (AR), an inflammation of the nasal mucous membranes, is induced through immunoglobulin $\mathrm{E}$ (IgE)-mediated responses to aeroallergens [1, 2]. 
The condition is manifested by sudden and recurrent nasal congestion, itchiness, sneezing, and runny nose [3]. AR is prevalent across the globe, [4-6] it is estimated that nearly $10-20 \%$ of the global population suffers from the disease [7], especially in China [8-10]. In the US, the disease reportedly affects up to 60 million people annually, with self-reported rates at $10-30 \%$ in adults and $40 \%$ in children [7]. In addition, the US reports annual direct health expenditures range of between $\$ 2$ and 5 billion. AR negatively affects the quality of life and results in reduced work productivity, [11-14] thus is considered a serious threat to the way of life globally.

Treatment therapies for AR and/or asthma comprise the use of several conventional medicines (CM) such as antihistamines, bronchodilators, and glucocorticoids [15]. However, the efficacy of these medications is not significant. Consequently, safer and more effective therapies are required to ensure effective management of the conditions. Apart from the aforementioned pharmacological methods, guidelines for clinical practice for AR stipulate that clinicians can administer non-pharmaceutical therapy treatment to patients $[16,17]$ and that 1 in every 5 people will always choose acupuncture [18]. Numerous guidelines have recommended acupuncture for managing AR $[13,18,19]$. Of note, this therapy has a long history in China and has successfully been used to manage AR symptoms [20-22]. Some studies reported that acupuncture achieved similar medicinal efficacy in treating moderate/severe AR, and the approach was safe without any apparent adverse reactions.[23, 24]. Further, numerous studies have shown that neuroendocrine immunity (NEI) plays a important role in AR $[25,26]$ and acupuncture may affect the function of the NEI system to treat AR. [13, 27-29].

In the recent past, an increasing number and size of random controlled trials (RCTs) for AR have been conducted with several systematic reviews [20,21, 30-33], confirming the efficacy and safety of acupuncture. However, these studies have only focused on a single acupuncture therapy against a control treatment for AR. Additionally, the systematic reviews only considered the cumulative effects of all the acupuncture treatments. Most articles only report on the evidence obtained upon comparing acupuncture methods with drugs or placebo acupuncture methods, however, they do not compare results across all existing acupuncture methods included in systematic reviews, such as manual acupuncture (MA), moxibustion (Mox), electronic acupuncture (EA), warm acupuncture (WA), acupuncture-moxibustion (AM), and fire acupuncture (FA). There is a need, therefore, to identify the optimal acupuncture methods for AR treatment.

Using the Bayesian network meta-analysis (NMA), we compared and rank the efficacy and safety of all acupuncture therapies adopted in AR treatment. Our findings provide credible evidence for the use of acupuncture therapies and elucidate the current controversies surrounding the approaches for their effective application in clinical operations as well as guiding health policies.

\section{Methods}

The systematic review is registered on PROSPERO, under the number CRD42020156200, in accordance with the Preferred Reporting Items for Systematic Reviews and Meta-Analyses (PRISMA-NMA) [34], and the accompanying checklist.

\section{Eligibility criteria and exclusion criteria Types of studies}

All articles reporting on RCTs and published in English/ Chinese, without any regional and publication restrictions, were included. The first period of the randomized cross-over trials was applied. Conversely, non-randomized clinical studies, quasi and cluster RCTs, case reports as well as studies where no data are available were excluded.

\section{Types of participants}

All patients, either male or female, and across all ages, who were diagnosed with AR were included. Similarly, participants with all types of AR (including intermittent $A R$ and persistent AR) regardless of their gender, etiology, ethnic group, severity, or diagnosed with specific criteria (such as mentioning any one of the criteria for the diagnosis of AR) met the inclusion criteria. Patients that diagnosed rhinitis which cannot be clearly attributed to allergic disorder would be excluded.

\section{Types of intervention}

Here, we included studies reporting on the use of acupuncture as monotherapy or additional therapies. The expected acupuncture approaches include MA, Mox, EA, AM, WA, and FA among others. Moreover, articles describing combinations of these acupuncture methods with $\mathrm{CM}$, regardless of needling technique or acupoint selections, were included, the primary acupuncture methods were mentioned. Besides, we did not include acupoint embedding, application, and injection, as well as bee venom acupuncture and other treatments because they used related drugs. Studies that integrated acupuncture with blood-letting therapy, cupping, and herbal medicine, were excluded from the analysis.

\section{Type of control group}

Different acupuncture therapies formed the basis for the control group and included both a placebo group (sham 
acupuncture (SA)) as well as a conventional-based medicine group.

\section{Types of outcome measures}

We included studies that covered one or more of the below-highlighted outcomes. Our systematic review primarily aimed to compare and rank the efficacy and safety of all acupuncture methods used in AR treatment. Based on this, the primary acceptable outcomes were nasal symptoms of AR, as numerous previous studies have reported the use of total nasal symptom score (TNSS). Secondary outcomes were analyzed as follows: (1) Quality of life was measured using rhinoconjunctivitis quality of life questionnaire (RQLQ); (2) IgE concentration was used to assess serum allergen-specific IgE; (3) Safety measures were assessed by describing adverse events (AEs) directly connected to the intervention. Outcome measures that were not relevant to AR would be excluded.

\section{Search strategy}

To retrieve relevant articles, we searched the databases including CBM, CNKI, WF, VIP, Web of Science (WOS), Embase, PubMed, and Cochrane Library, from their inception to 1st July 2020. Additionally, other resources such as clinical trial registries (WHO ICTRP, Clinical Trials, and ChiCTR) and Allied and Complementary Medicine Database (AMED) were used to reduce publication bias in data. Additional trials, including relevant systematic reviews and meta-analyses, were identified according to the list of all identified publications. Since different databases required different retrieval methods, our search terms covered three groups: (1) Clinical conditions namely AR, Allergic Rhinitis, and, rhinallergosis; (2) acupuncture methods such as acupuncture therapy, manual acupuncture, moxibustion, electroacupuncture, and fire acupuncture, etc.; (3) study types such as randomized controlled trials. The mode of retrieval used was dependent on subject terms and free words, and these terms distinguished between published studies. We used "and", "or" to connect the name of terms. According to PubMed's search strategy (Table 1), Chinese and English retrieval modes were expected to be similar.

\section{Study selection and data extraction}

First, all reviewers were professionally trained to master the review process. To select the studies, the reviewers first read the title/abstract of the review to identify duplicate studies, then, they uploaded eligible articles to a database built using NoteExpress V.3.0. Further, the two reviewers (GG and GX) independently schemed through the titles, abstracts, and keywords. The two reviewers solved any disagreements through a consensus, following a discussion. However, whenever the discussion was conflicting, a third reviewer (LZ) would help in making a final decision.

The aforementioned reviewers independently extracted data using a standardized eligibility form, and in case of a disagreement, a third reviewer (LZ) came in. Any missing information was obtained by contacting the corresponding author of the specific article. General data from the selected studies including, the name of the first author, year of publication, country, study design, sample size, intervention group, control group, outcome, results, were extracted and recorded into an Excel sheet. Also, the reviewers independently measured the risk of bias in the included studies following the guidelines of the Cochrane Handbook V.5.3.0. In case of insufficient or ambiguous data, one reviewer contacted the corresponding author of the articles, requesting for additional details. However, if the details were not available, a description was added to the final report. A summary of the selection procedures is outlined using the PRISMA flow chart.

Table 1 Search strategy (through PubMed)

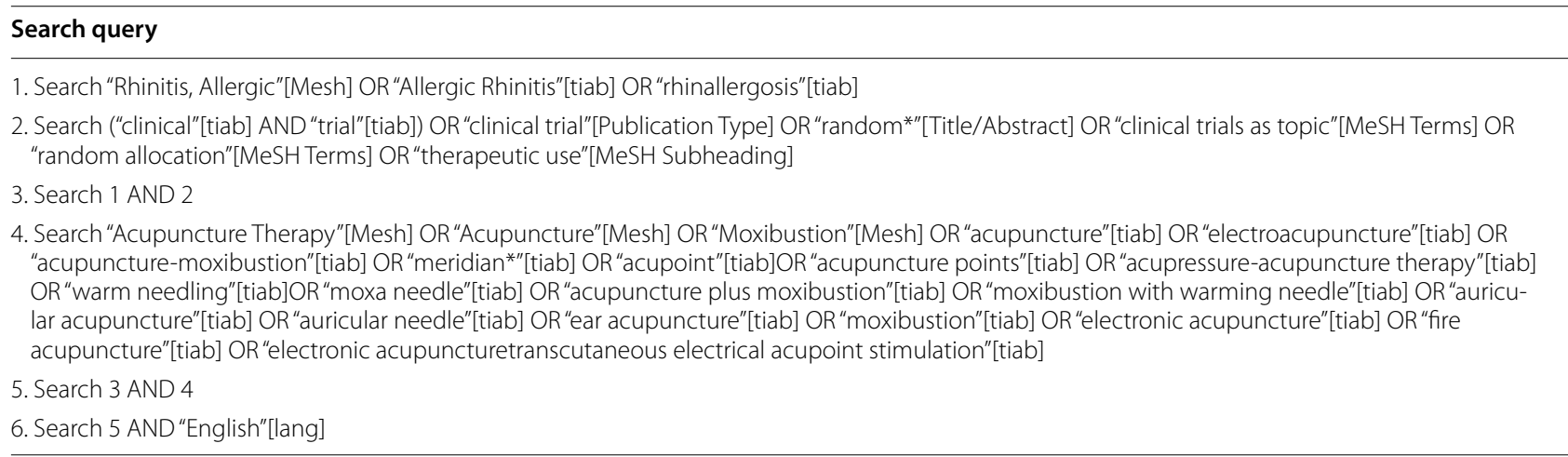




\section{Study quality assessment}

Here, 2 reviewers evaluated the risk of bias in all included RCTs using the Cochrane Collaboration Risk of Bias Tool [35]. This approach comprised sequence generation, allocation concealment, blinding of participants and outcome assessors, incomplete outcome data, selective outcome reporting, as well as other sources of bias. The findings were used to rank risk levels as low, unclear, or high. In case of any disagreement, the third reviewer (LZ) was consulted to generate a consensus. Review Manager (RevMan, version 5.3, the Nordic Cochrane Center, the Cochrane Collaboration, 2012 Copenhagen, Denmark) was used to generate the figure highlighting the risk of bias.

\section{Pairwise meta-analysis}

Revman was used to analyze the data. Pre-post differences were used as outcome indicators for each included study. Further, 3-arm RCTs were separated into two arms for all possible combinations in the meta-analysis. The fixed-effects model utilized the Mantel-Haenszel procedure, otherwise, the random-effects model adopted by Der Simonian-Laired procedure was used. The $\mathrm{I}^{2}$ statistic and $p$ value were used to identify and measure the heterogeneity among the studies. All data were analyzed with a 95\% confidence interval (CI). For continuous data, the standard mean differences (SMD) were used. According to the Cochrane Handbook, when $p>0.05, \mathrm{I}^{2}<50 \%$, we considered that no heterogeneity existed.

\section{Network meta-analysis}

To compare the effects of different acupuncture treatments, a Bayesian network analysis was performed [36] using the Aggregate Data Drug Information System (ADDIS V.1.16.8, Drugis, Groningen, NL), with Markov Chain Monte Carlo (MCMC) method [37]. The parameters were set at 4 chains for simulation, while the simulation iterations were set to 50,000. First, we performed 20,000 adjustment iterations to eliminate the effect of the initial value, then, integrated indirect and direct evidence from all the RCTs according to the node splitting method. Meanwhile, STATA software Version.15.0 (Stata Corp LP, College Station, Texas, USA) was used to generate plots of the network meta-analysis and compare each outcome. Finally, we generated figure ranking probabilities for all the interventions, after which local inconsistency was assessed using the node-splitting method. Generally, all nodes showed P-values greater than 0.05 in inconsistency tests, implying that no significant statistical difference existed between direct and indirect comparisons. Potential scale reduced factor (PSRF) reflected the convergence of the model, with a PSRF of 0 and $100 \%$ indicating the worst and best treatments, respectively.

\section{Publication bias}

We performed a funnel plot indicating digital-based modeling of the results to eliminate reporting data with bias.

\section{Assessing reviewer agreement}

We performed the intra-class correlation coefficient (ICC) to evaluate the consistency of two reviewers. Briefly, the two authors independently evaluated the quality of RCTs. The composite ICC score value was 0.93 .

\section{Results}

Study selection

After the primary search process, we identified 3004 potentially relevant studies from these databases. After eliminating 1132 duplicates, 1872 articles were retained, from which only 64 remained after reading through the titles and abstracts. When we did a full-text assessment, 25 articles were excluded. Eventually, a total of 39 RCTs were included [38-76] in this systematic review (Fig. 1).

\section{Study characteristics}

In total, 39 studies were included in the final Bayesian meta-analysis, 37 RCTs [38-71, 73, 75, 76] were published in Chinese while and 2 RCTs [72, 74] were written in English. All the 39 articles were published between 2006 and 2020, with 3433 participants. The interventions in these studies included MA, EA, WA, AM, FA, Mox, $\mathrm{SA}, \mathrm{CM}$, and the combinations between these methods or with CM. Besides, the mean patient age of 26 to 45 years (except that 7 RCTs didn't specify clear age [38, $48,52,64,69,75,76])$. The sample size of the included RCTs ranged from 37 to 422 . Group allocation was primarily performed using a 1:1 allocation ratio. And the ratio of male to female is basically the similar. The selection of acupoint in the included acupuncture treatments was diverse, the main choices included Xinwu point (Sphenopalatine Ganglion), Yingxiang (LI 20), Yintang (DU 29), Shangyingxiang point, Feishu (BL 13), Dazhui (DU 14), Hegu (LI4). In these studies, treatment duration ranged from 4 to 8 weeks. A reduction in the RQLQ score was, in most cases the mentioned outcome measures. Detailed information and findings of all included studies are displayed in Table 2.

\section{Study quality assessment}

Herein, we assessed the risk of bias using the Cochrane Handbook Version 5.3. Meanwhile, the results were summarized and analyzed using RevMan 5.3. Overall, there were 4 [60, 72-74] high-quality RCTs in the included studies, but no case about high risk was reported. All RCTs were randomized, however, 8 trials $[38,51,52,54,58,61,75,76]$ did not use random 


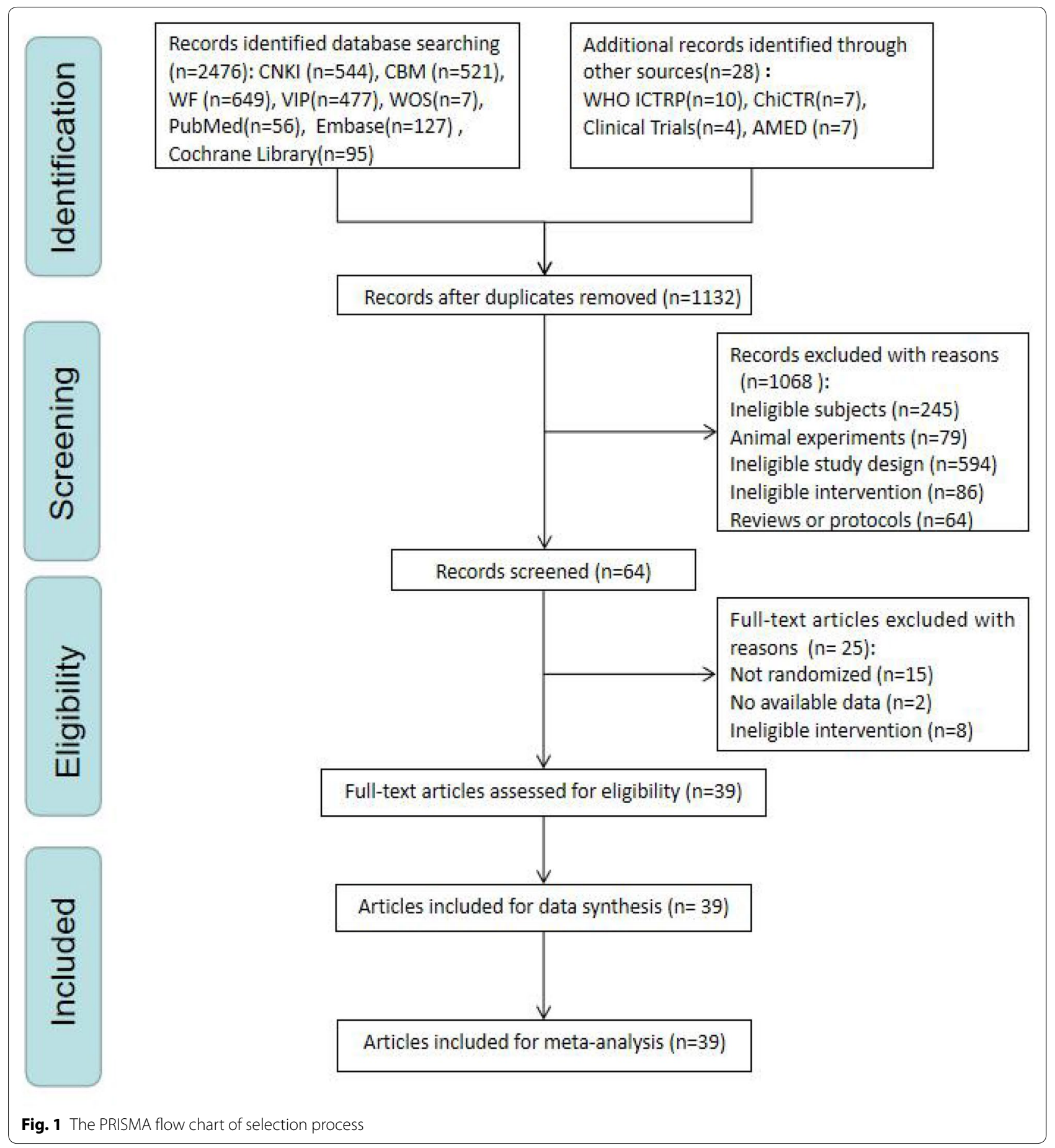

sequence generation. Notably, 12 studies [44, 45, 53, $57,59,60,68,70-74]$ showed low risk of allocation concealment. The blinding method was performed in 5 studies [45, 60, 72-74], and all of them blinded outcome assessors, further, 4 RCTs [60, 72-74] showed that they blinded the participants. Data on the outcome from all the 5 studies were complete and ranked as low risk. In selective outcome reporting, 38 RCTs were evaluated as low risk, but one RCT [49] as unclear because of insufficient information. In the other bias, 14 trials $[38,39,43,46,47,52,54,56,60,63,65,69,75$, 76 ] were unclear as they lacked adequate information. The methodological quality assessment results for each included study are displayed in Fig. 2. 


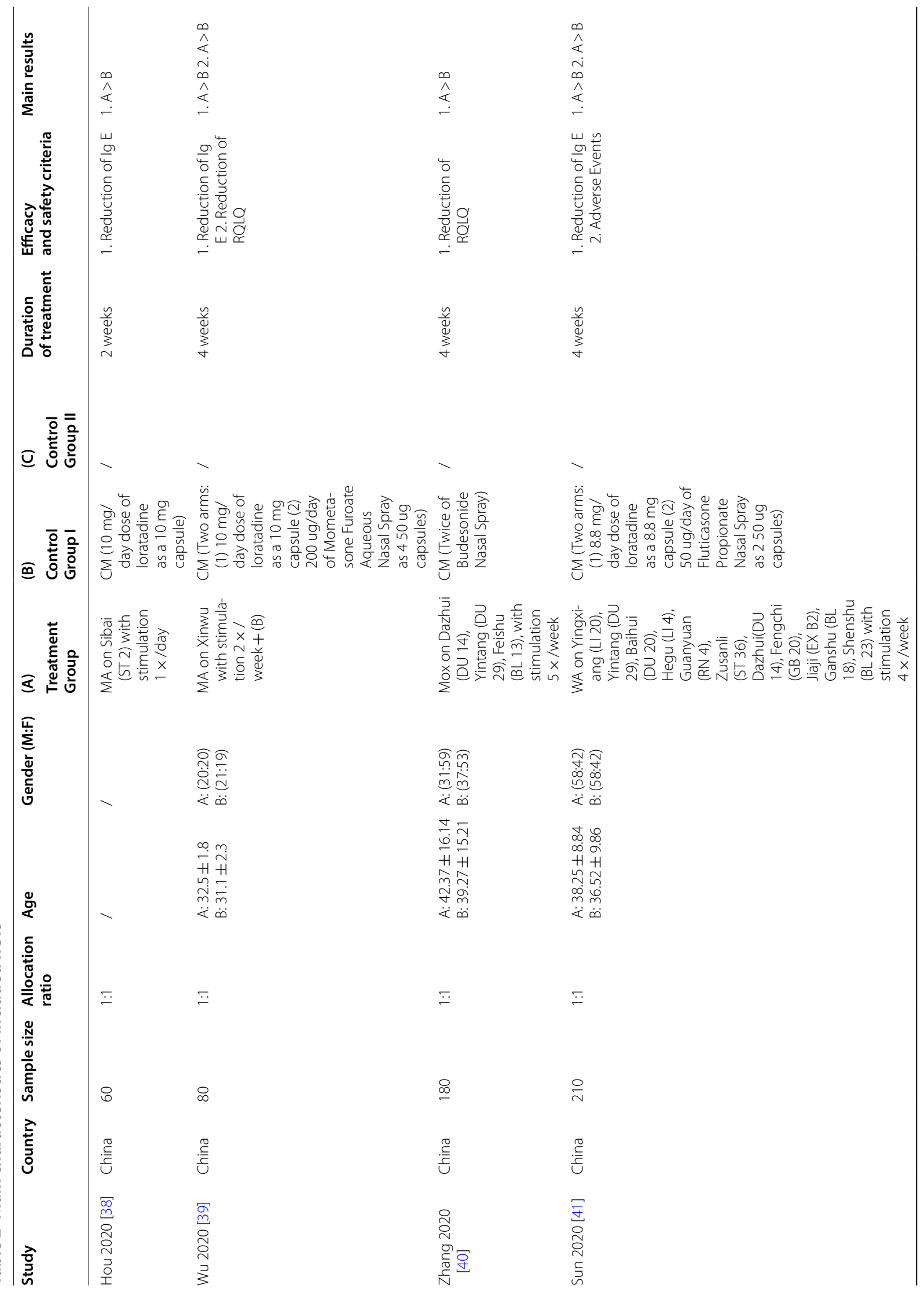




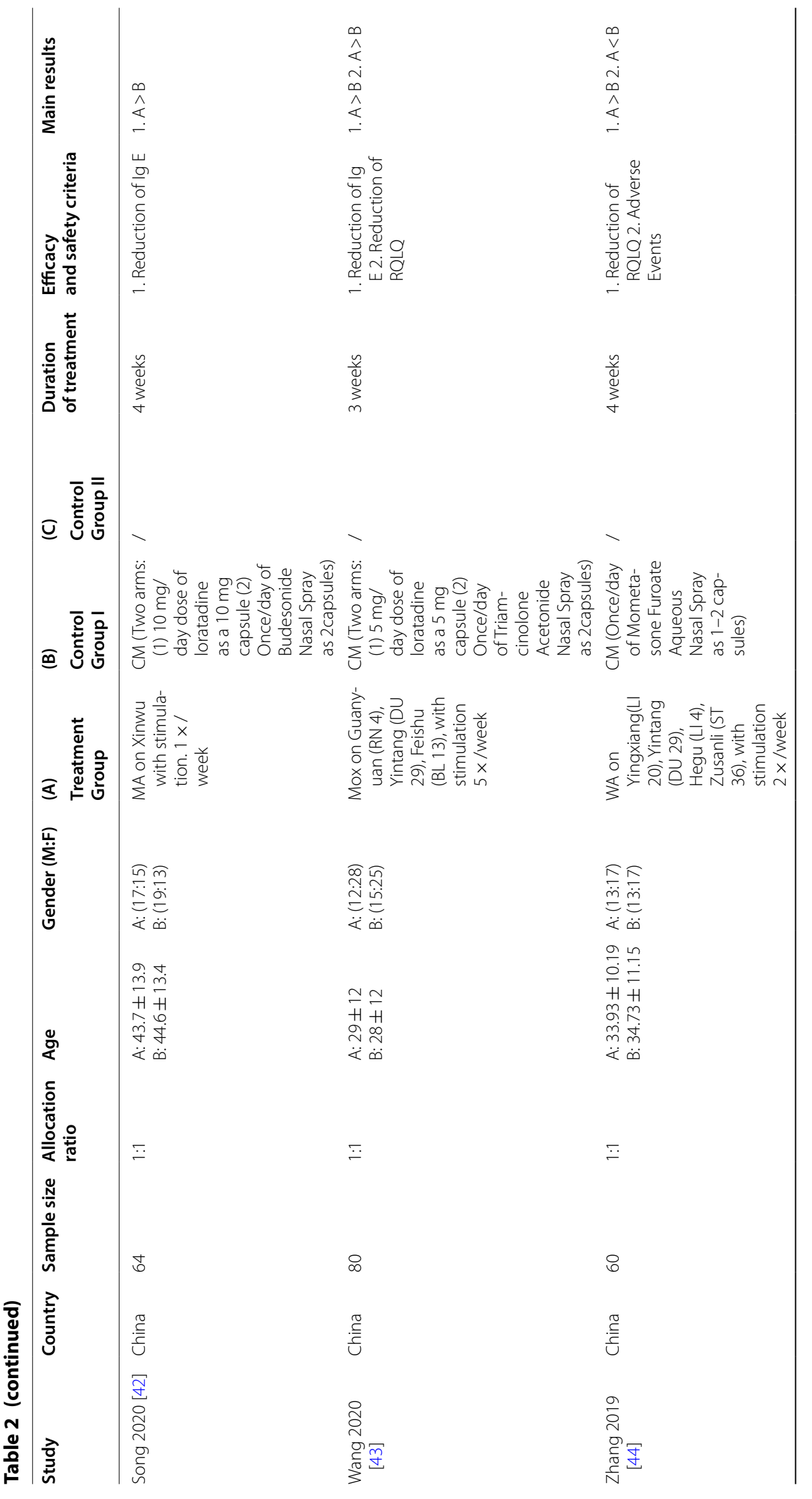




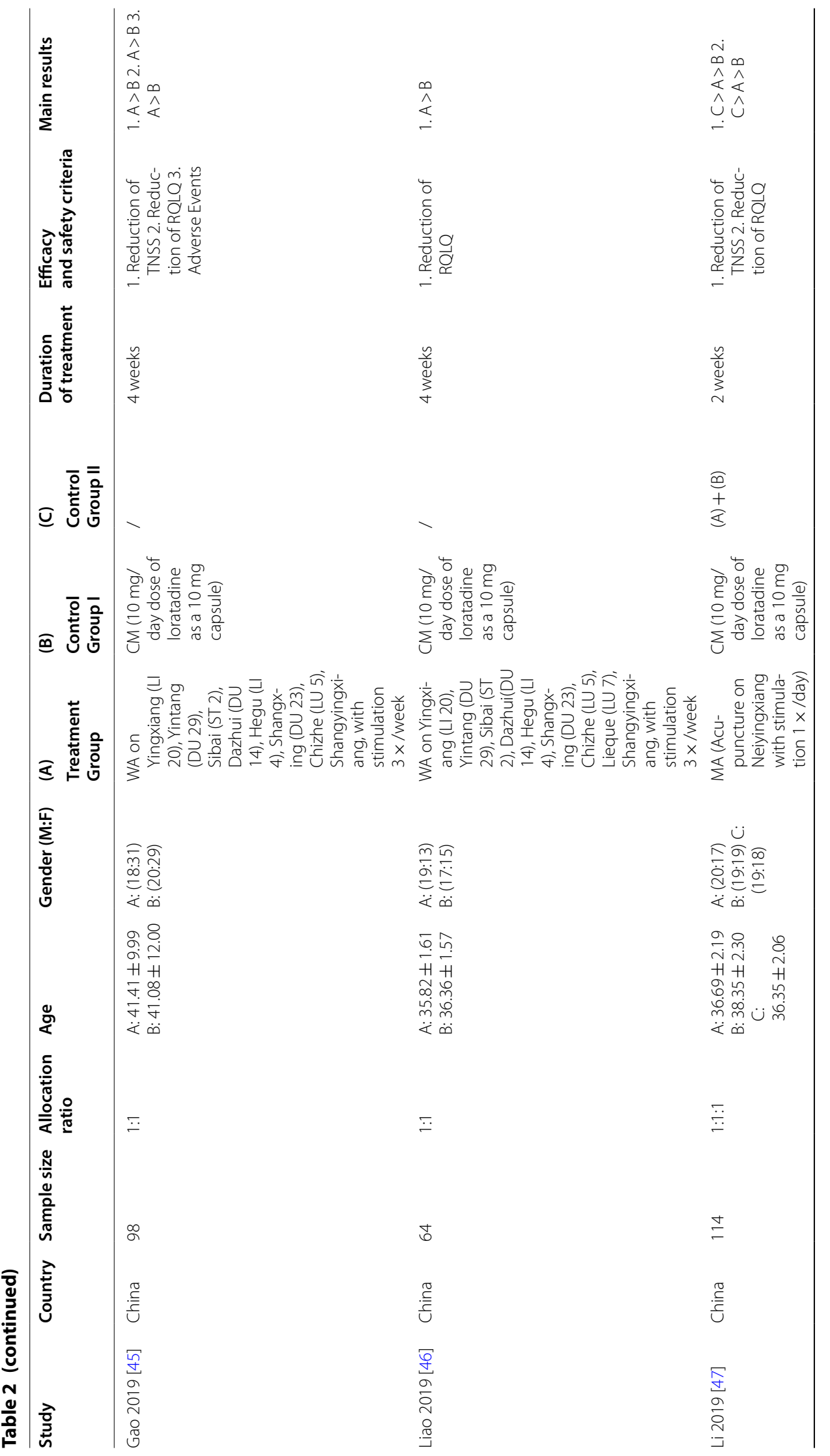




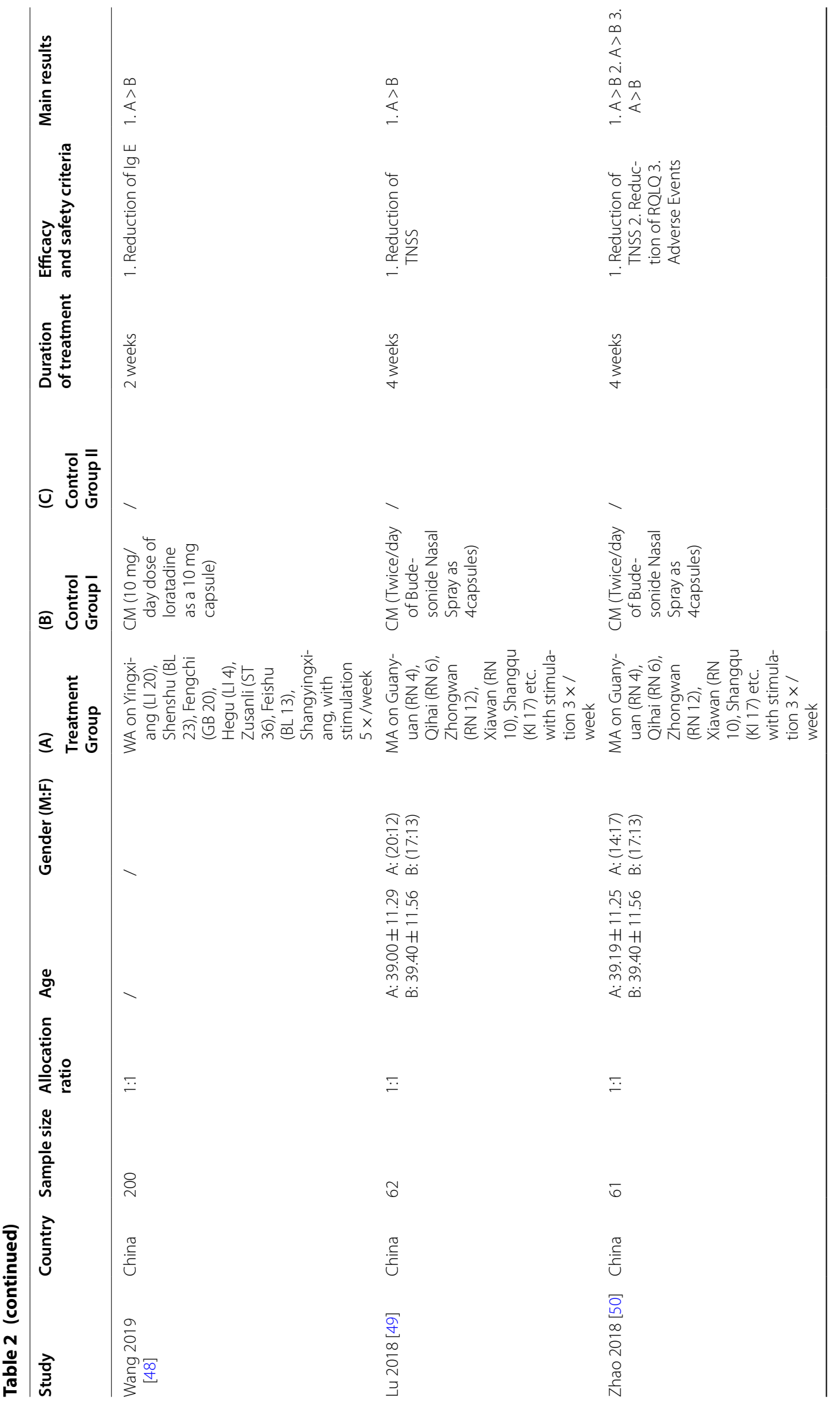




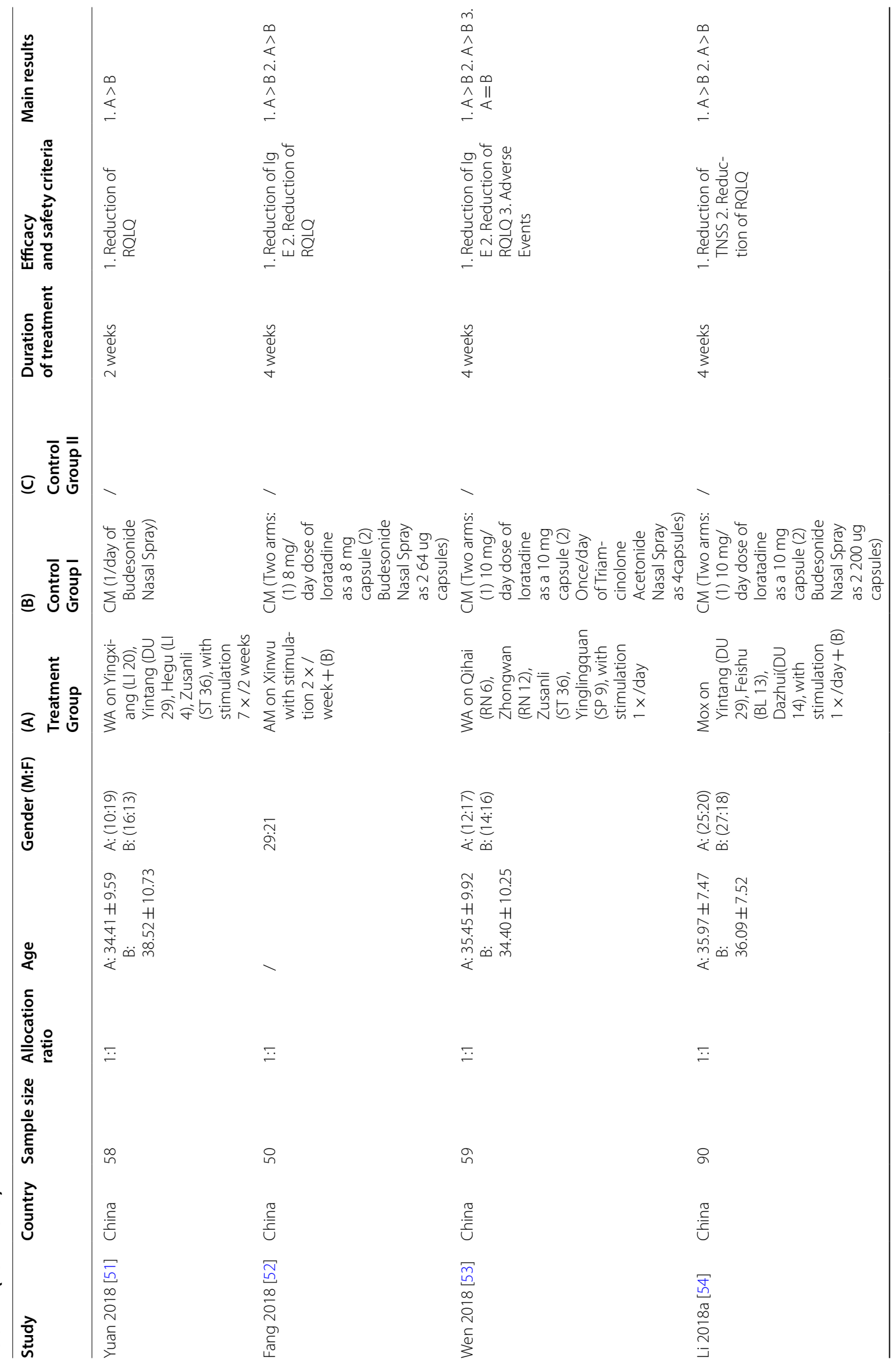




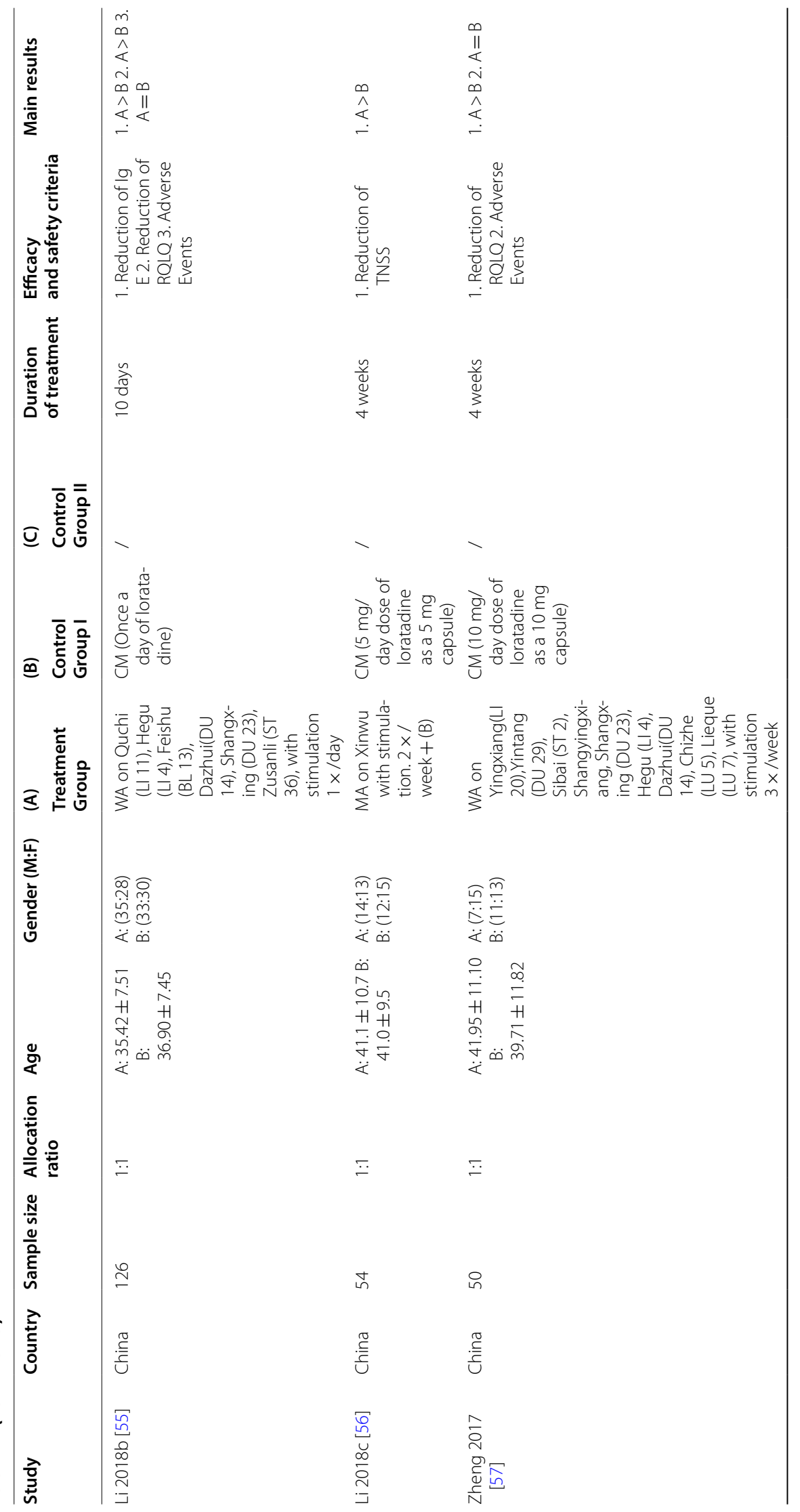




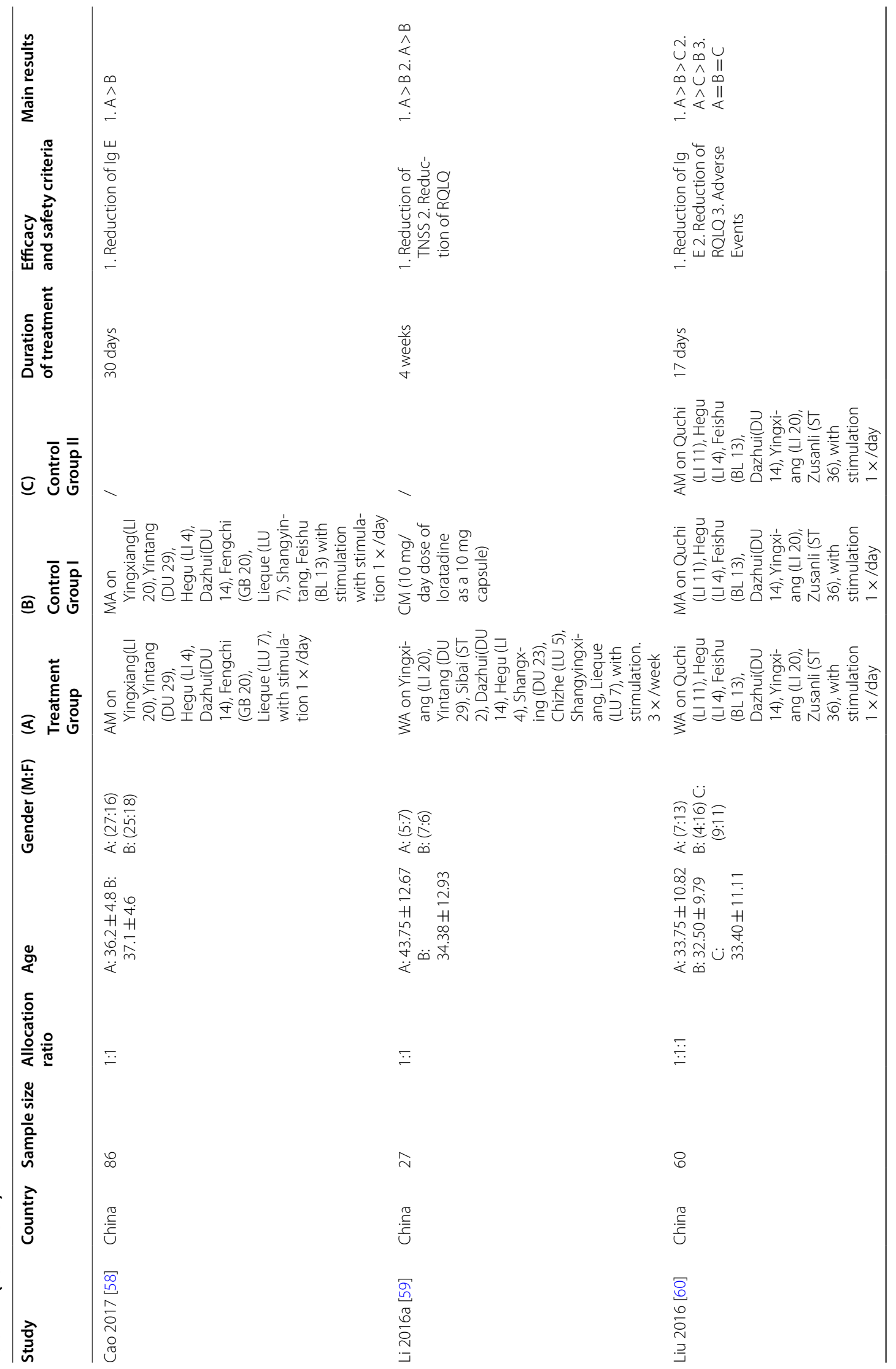




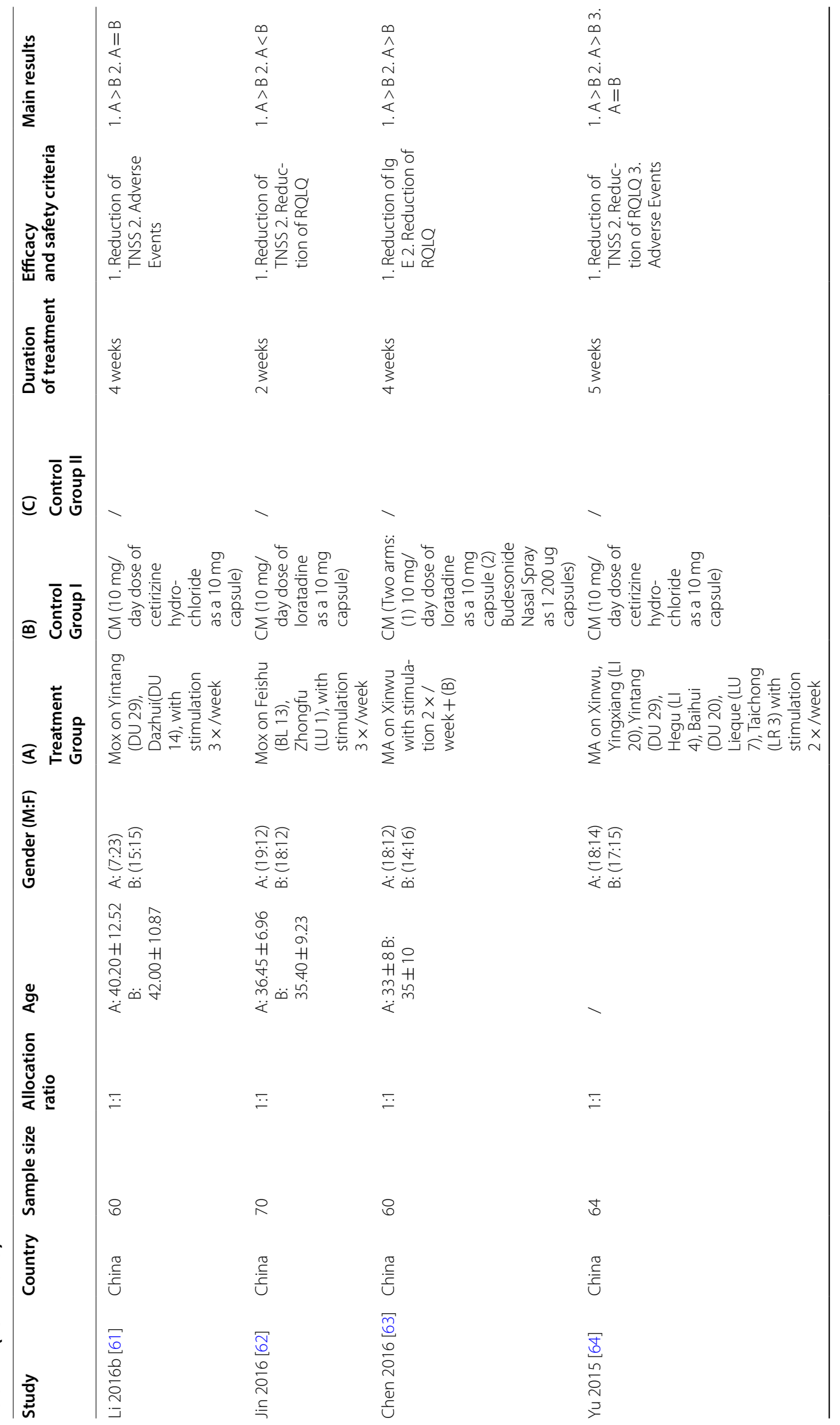




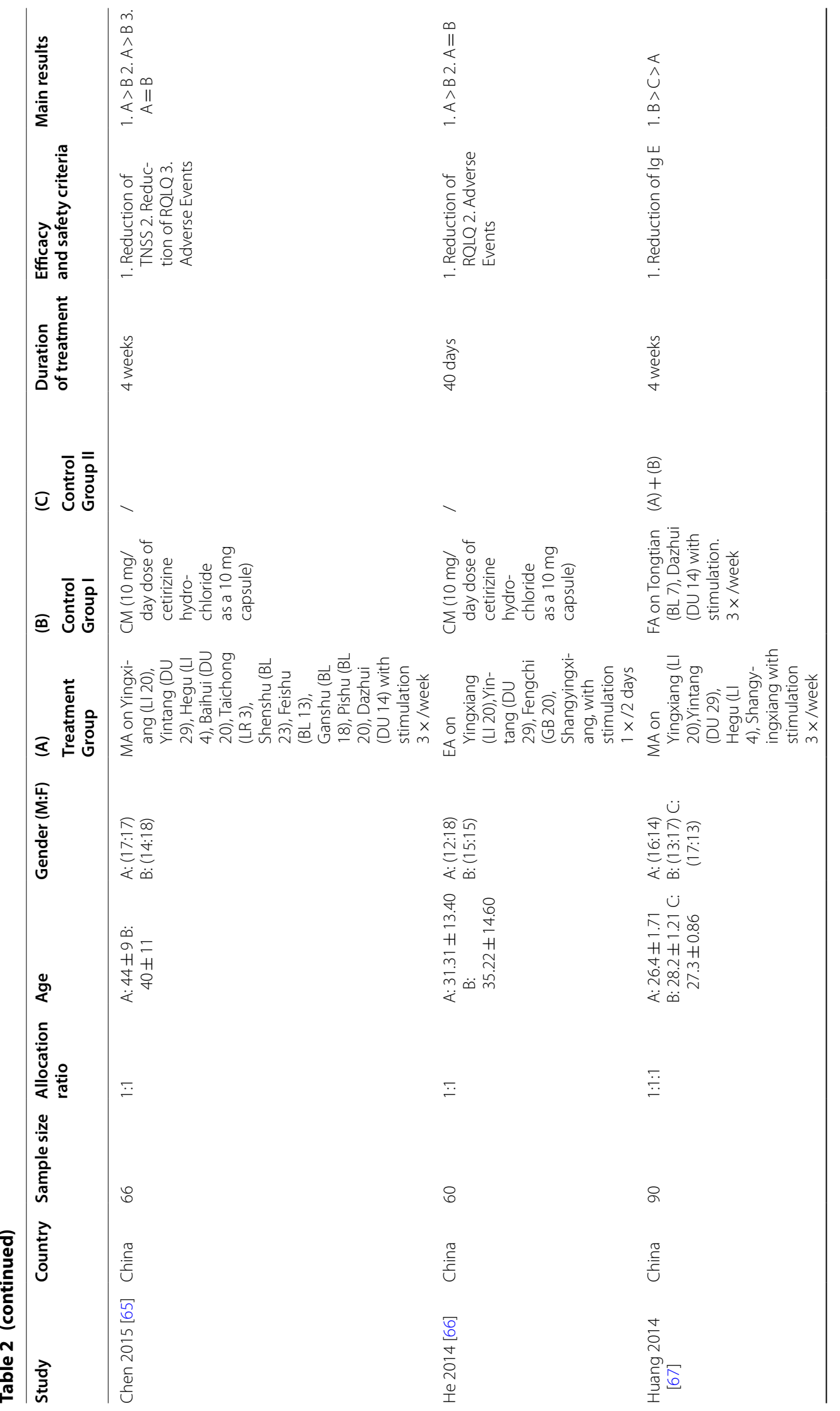




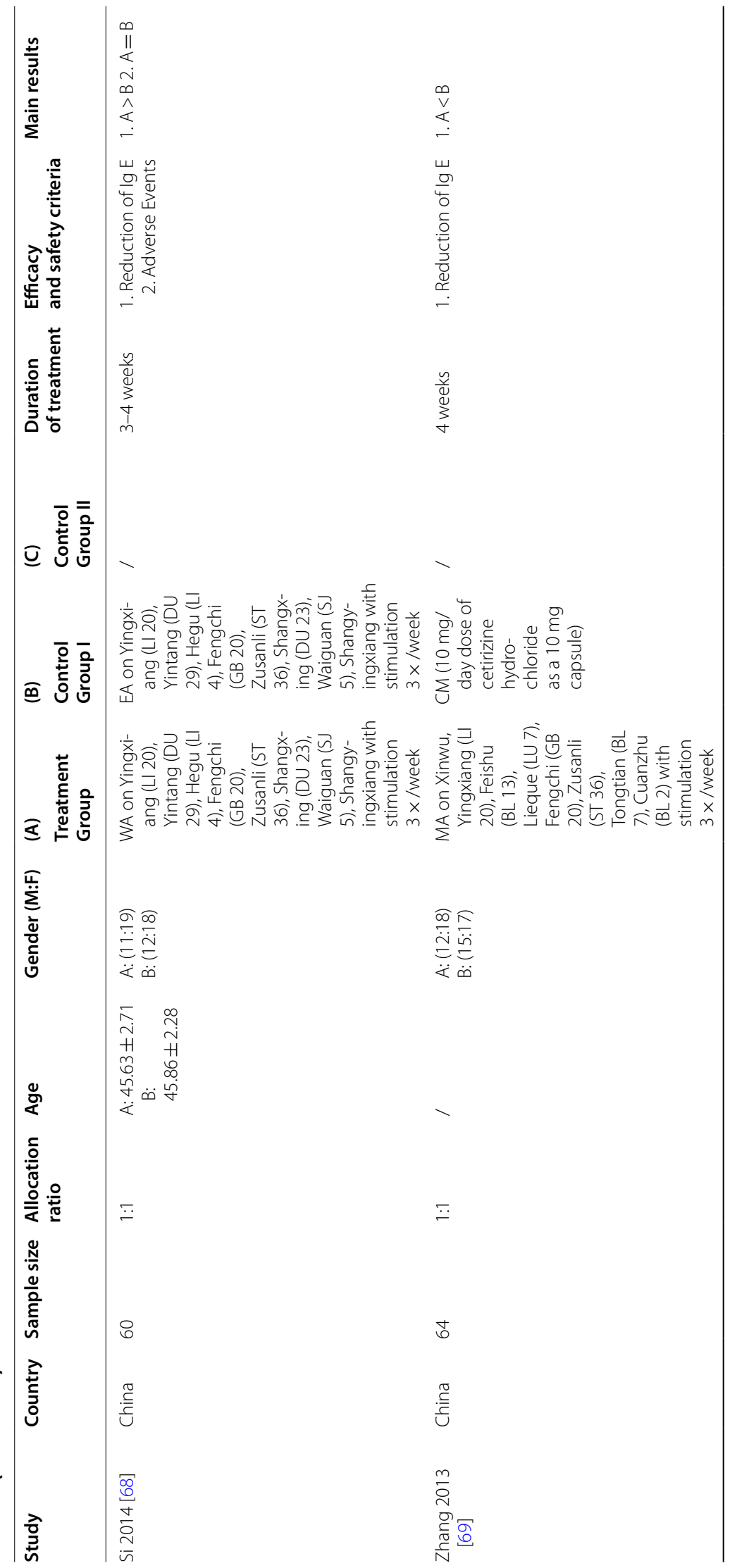




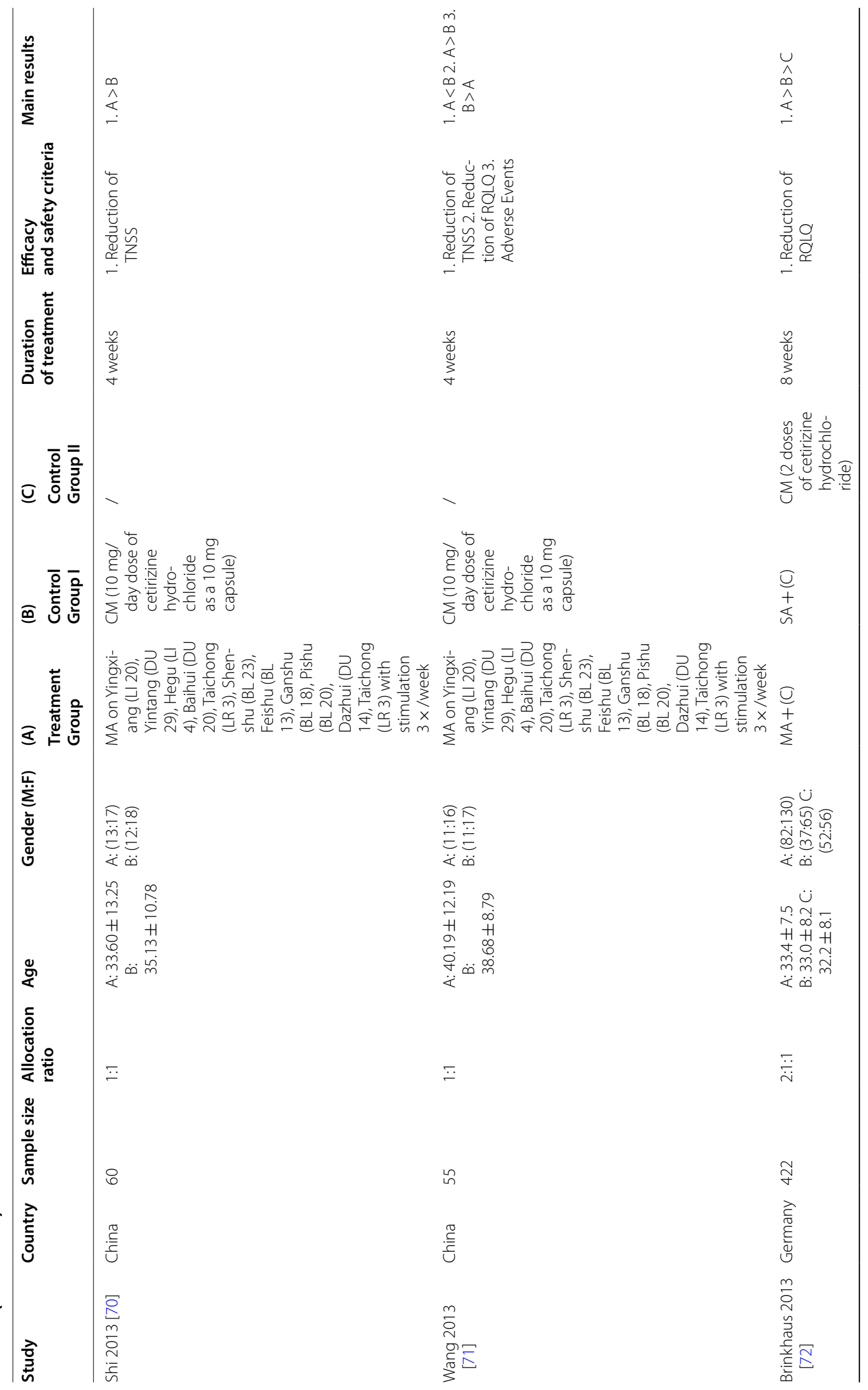




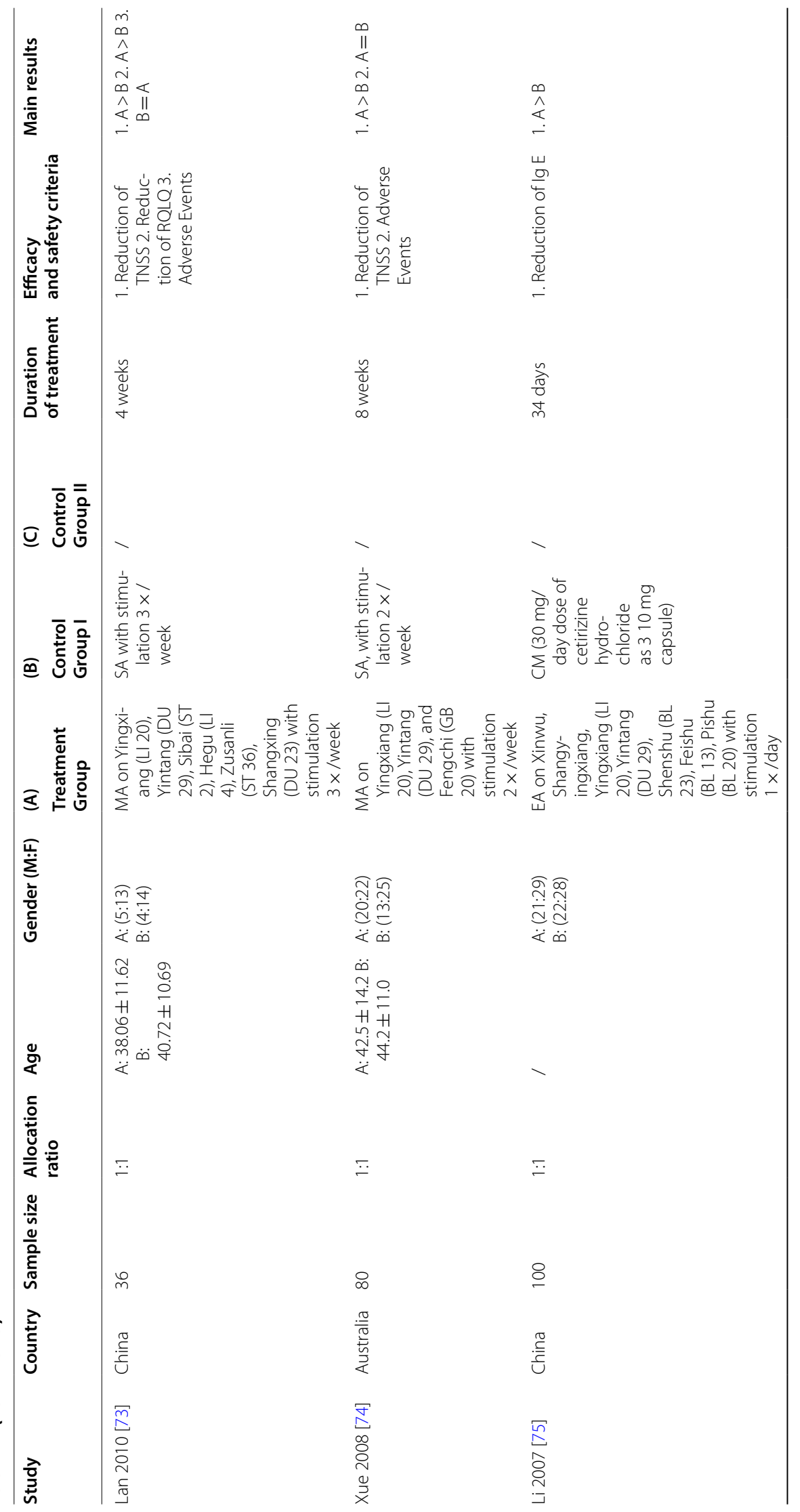




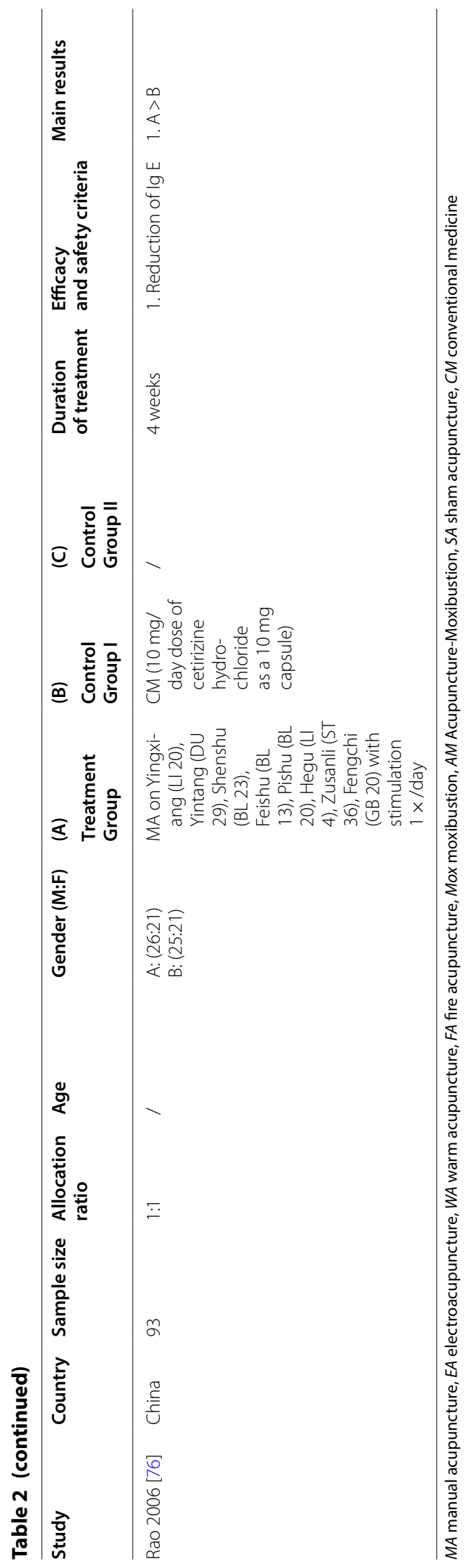




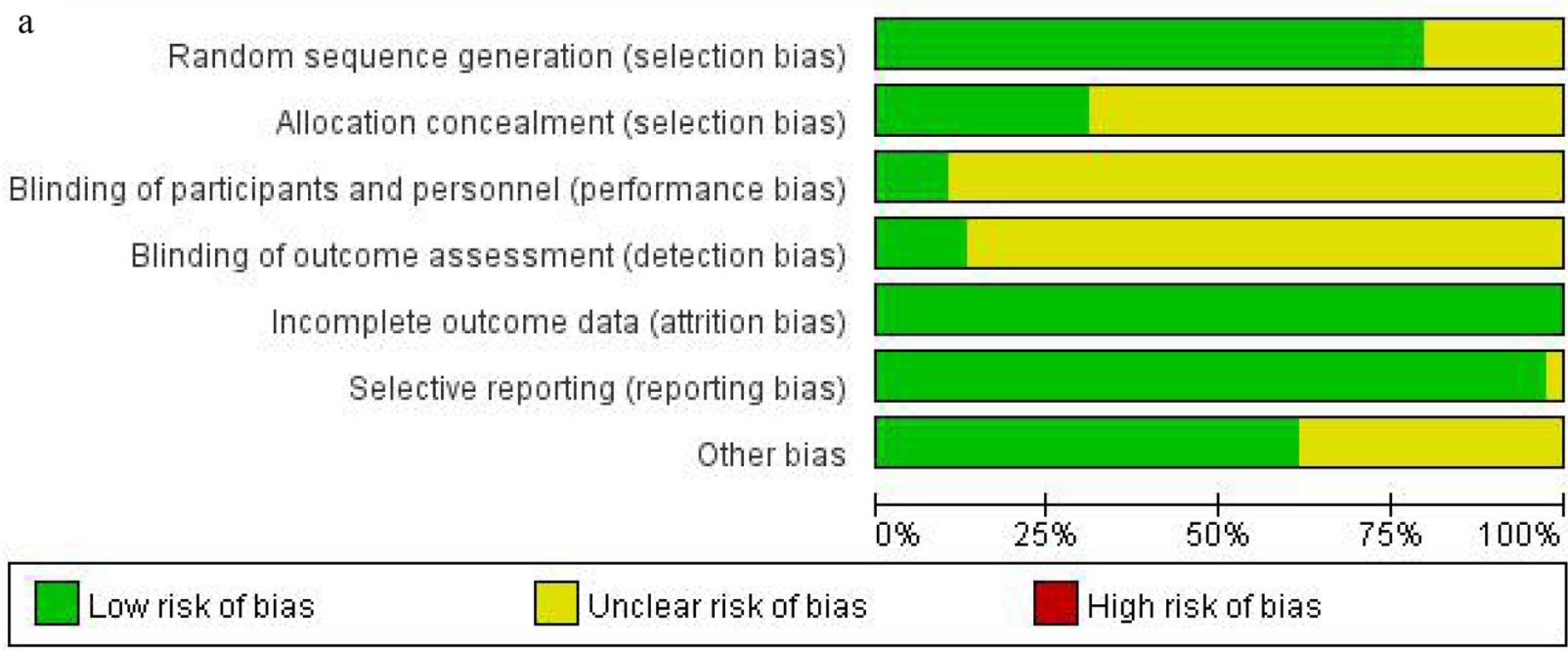

Fig. 2 a Risk of bias graph; $\mathbf{b}$ Risk of bias summary

\section{Pairwise meta-analysis results Reduction in TNSS}

We performed 6 pairwise meta-analyses to compare the effectiveness of different acupuncture methods with CM. Detailed results are shown in Table 3. Mox and CM showed statistically significant differences (3 RCTs, SMD, 1.03; 95\% CI 0.37 to 1.09 ); MA + CM was highly effective in improving TNSS compared to CM (1 RCTs, SMD, 3.30; 95\% CI 2.29 to 4.31) and MA (1 RCTs, SMD, 1.47 ; 95\% CI 0.74 to 2.21 ). No significant difference between MA and CM, WA and CM, MA and SA were noted.

\section{Reduction in RQLQ}

Here, 13 pairwise meta-analyses were generated to compare the effectiveness of different acupuncture treatments with CM. Detailed results are shown in Table 4. MA + CM and MA showed statistically significant differences (1 RCT, SMD, $-1.92 ; 95 \%$ CI -1.60 to $-0.24)$; $C M$ and MA + CM displayed significant differences (3 RCTs, SMD, -2.06 ; 95\% CI -2.42 to -1.71 ); Statistically significant differences (1 RCT, SMD, 1.66; 95\% CI 1.28 to 2.04) were reported between MA + CM and $\mathrm{SA}+\mathrm{CM} ; \mathrm{CM}$ and $\mathrm{SA}+\mathrm{CM}$ displayed significant differences (1 RCT, SMD, -0.66 ; $95 \%$ CI -1.06 to $-0.27) ; \mathrm{MA}$ and SA displayed significant differences (1 RCT, SMD, 0.92 ; $95 \%$ CI 0.23 to 1.61 ); Statistically significant differences (8 RCTs, SMD, 1.71; 95\% CI 0.87 to 2.56) were reported between WA and CM; CM and AM showed statistically significant differences (1 RCT, SMD, -1.87 ; $95 \%$ CI -2.57 to -1.22 ). No significant difference was noted between MA and CM, MA and WA, MA and AM, WA and AM, Mox and CM, CM and EA.

\section{Reduction in $\lg E$}

We generated 13 pairwise meta-analyses to compare the effectiveness of different interventions. Detailed results are highlighted in Table 5. CM and MA+CM showed statistically significant differences (1 RCT, SMD, -0.55 ; $95 \%$ CI -1.00 to -0.11 ). However, no significant difference was reported in others.

\section{Network meta-analysis results Network plot for different interventions}

In Fig. 3, the thickness of the line is positively correlated with the two intervention methods, whereas the size of the points is proportional to the weight of the sample size in the intervention. A total of 15 studies covering 6 interventions and 995 participants were merged for metaanalysis of the reduction in TNSS (Fig. 3a). Reduction of RQLQ was reported in 24 RCTs including 2,080 patients and 9 methods (Fig. 3b), whereas reduction of Ig E was revealed in 17 studies involving 1,498 participants and 9 therapies (Fig. 3c).

\section{Evaluating statistical inconsistency}

All local inconsistency tests were performed with the node-splitting method. Results of the inconsistency test on the reduction of RQLQ and Ig E (P>0.05) demonstrated that no significant difference existed between direct and indirect comparisons. Furthermore, no inconsistency of the model was found when the node-splitting 
b

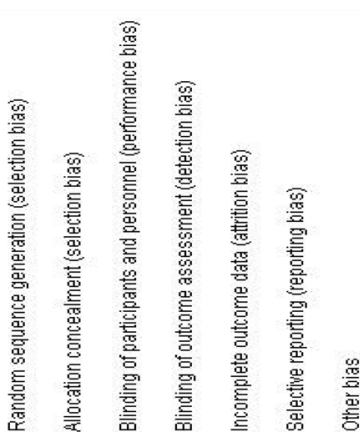

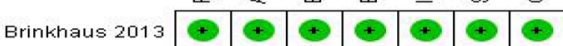

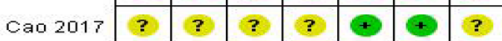

\begin{tabular}{ll|l|l|l|l|c|c|} 
chen 2015 & 9 & $?$ & $?$ & $?$ & 9 & ( & 9 \\
\hline
\end{tabular}

\begin{tabular}{l|l|l|l|l|l|l|l|}
\hline Chen 2016 & $\oplus$ & $?$ & $?$ & $?$ & $\oplus$ & $\oplus$ & $?$ \\
\hline
\end{tabular}

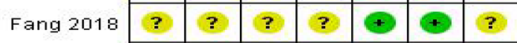

Gao $2019 \oplus \odot ?+\odot \oplus$

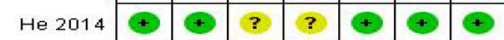

\begin{tabular}{ll|l|l|l|l|l|l|}
$\operatorname{Hou} 2020$ & $?$ & $?$ & $?$ & $?$ & 9 & 9 & $?$
\end{tabular}

Huang 2014 (4)

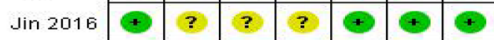

Lan $2010 \odot \oplus \odot \oplus \odot \oplus$

Li 2007 ?

Li 2016a 9 (6) 3 ? 9

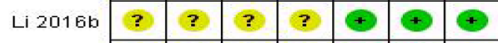

\begin{tabular}{l|l|l|l|l|l|l|l|} 
Li 2018a & $?$ & $?$ & $?$ & $?$ & $\oplus$ & $\odot$ & $?$ \\
\hline
\end{tabular}

Li 2018b $\odot$ ค

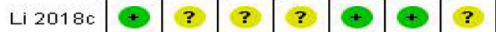

Li 2019 (4)

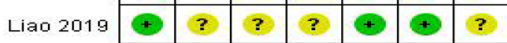

Liu 2016 $\odot$ ค

Lu 2018 $\odot$ ?

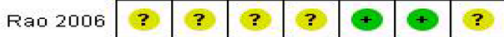

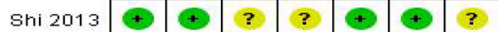

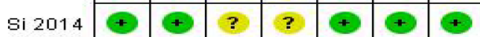

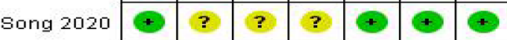

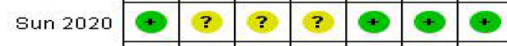

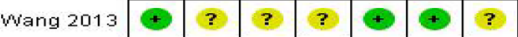

Wang $2019 \odot ? ?$

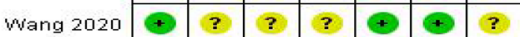

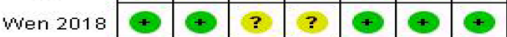

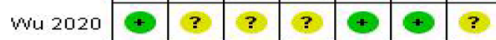

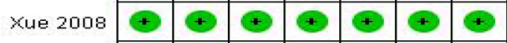

Yu $2015 \odot ? ?$

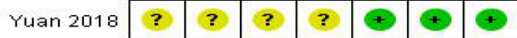

Zhang 2013 (4)

Zhang $2019 \odot \odot \overbrace{}^{\circ}+? \odot \odot$

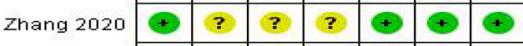

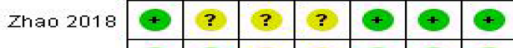

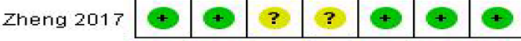

Fig. 2 continued 
Table 3 Pairwise meta-analysis of of reduction of TNSS

\begin{tabular}{llclc}
\hline Comparison & Number & SMD $(\mathbf{9 5 \%} \mathbf{C l})$ & $\mathbf{I}^{\mathbf{2}}(\%)$ & $\boldsymbol{p}$ \\
\hline MA VS CM & 8 & $0.28(-0.25,0.81)$ & 87 & $<0.00001$ \\
WA VS CM & 2 & $0.12(-0.19,1.33)$ & 87 & 0.006 \\
Mox VS CM & 3 & $1.03(0.37,1.09)$ & 82 & 0.28 \\
CM VS MA + CM & 1 & $-3.30(-2.29,-4.31)$ & - & - \\
MA VS MA + CM & 1 & $-1.47(-0.74,-2.21)$ & - & - \\
MA VS SA & 2 & $1.05(-0.45,2.56)$ & 92 & 0.0003
\end{tabular}

Italic values indicate significant difference

MA manual acupuncture, WA warm acupuncture, Mox moxibustion, SA sham acupuncture, $C M$ conventional medicine

Table 4 Pairwise meta-analysis of of reduction of RQLQ

\begin{tabular}{llclc}
\hline Comparison & Number & SMD $(\mathbf{9 5} \% \mathrm{Cl})$ & $\mathbf{I}^{\mathbf{2}}(\%)$ & $\boldsymbol{p}$ \\
\hline MA VS WA & 1 & $-0.53(-1.43,0.36)$ & - & - \\
MA VS CM & 6 & $0.79(-0.05,1.63)$ & 92 & $<0.00001$ \\
MA VS MA + CM & 1 & $-1.92(-1.60,-0.24)$ & - & - \\
MA VS AM & 1 & $-0.13(-1.01,0.74)$ & - & - \\
MA VS SA & 1 & $0.92(0.23,1.61)$ & - & - \\
WA VS CM & 8 & $1.71(0.87,2.56)$ & 94 & $<0.00001$ \\
WA VS AM & 1 & $-0.50(-0.39,1.39)$ & - & - \\
Mox VS CM & 4 & $0.14(-0.37,0.64)$ & 84 & 0.0003 \\
CM VS MA + CM & 3 & $-2.06(-2.42,-1.71)$ & 25 & 0.27 \\
CM VS AM & 1 & $-1.87(-2.57,-1.22)$ & - & - \\
CM VS EA & 1 & $-0.13(-0.64,0.37)$ & - & - \\
CM VS SA + CM & 1 & $-0.66(-1.06,-0.27)$ & - & - \\
MA + CM VS & 1 & $1.66(1.28,2.04)$ & - & - \\
SA + CM & & & & \\
\hline
\end{tabular}

Italic values indicate significant difference

$M A$ manual acupuncture, $E A$ electroacupuncture, WA warm acupuncture, Mox moxibustion, $A M$ Acupuncture-Moxibustion, $S A$ sham acupuncture, $C M$ conventional medicine

method was used in TNSS, thus we selected the consistency model.

\section{Reduction in TNSS}

We conducted a network graph between the 6 treatments through STATA 15.0 (Fig. 3a). According to the PSRF results (value close to 1, Additional file 1: Appendix S1), we performed the network meta-analysis using the consistency model, and the figure of ranking probability was generated (Fig. 4a). Based on Fig. 4a, MA + CM, Mox, and WA treatments ranked the top 3 in this study. The Mox was recommended as the most effective intervention in reducing nasal symptoms out of the 6 treatments in this study. As highlighted in Table 6, 5 interventions $(\mathrm{MA}+\mathrm{CM}, \mathrm{Mox}, \mathrm{WA}, \mathrm{MA}, \mathrm{CM})$ were significantly more effective than SA.
Table 5 Pairwise meta-analysis of of reduction of $\lg \mathrm{E}$

\begin{tabular}{llccc}
\hline Comparison & Number & SMD $(95 \% \mathrm{CI})$ & $\mathbf{I}^{\mathbf{2}}(\%)$ & $\boldsymbol{p}$ \\
\hline MA VS CM & 5 & $0.06(-0.33,0.46)$ & 69 & 0.01 \\
MA VS WA & 1 & $-0.73(-2.47,1.02)$ & - & - \\
MA VS AM & 2 & $-0.31(-0.83,0.21)$ & 7 & 0.3 \\
MA VS FA & 1 & $-0.15(-0.87,0.57)$ & - & - \\
MA VS MA+FA & 1 & $-0.29(-1.01,0.43)$ & - & - \\
WA VS CM & 4 & $0.49(-0.25,1.22)$ & 94 & $<0.00001$ \\
WA VS AM & 1 & $1.34(-0.72,3.40)$ & - & - \\
WA VS EA & 1 & $-0.1(-0.61,0.41)$ & - & - \\
Mox VS CM & 1 & $0.33(-0.11,0.78)$ & - & - \\
CM VS MA + CM & 1 & $-0.55(-1.00,-0.11)$ & - & - \\
CM VS AM & 1 & $-0.55(-1.12,0.01)$ & - & - \\
CM VS EA & 1 & $-0.36(-0.77,0.02)$ & - & - \\
FA VS FA + AM & 1 & $-0.16(-0.87,0.56)$ & - & - \\
\hline
\end{tabular}

Italic value indicates significant difference

$M A$ manual acupuncture, $E A$ electroacupuncture, WA warm acupuncture, FA fire acupuncture, Mox moxibustion, $A M$ Acupuncture-Moxibustion, $C M$ conventional medicine

\section{Reduction in RQLQ}

Using STATA 15.0, we generated a network plot between the 9 interventions (Fig. 3b). Based on the PSRF (value close to 1, Additional file 1: Appendix S2 (a)) and P-values (Additional file 1: Appendix S2 (b)), Bayesian network meta-analysis was performed using the consistency model. The ranking probability figure was also generated (Fig. 4b). MA + CM, AM, and WA therapies ranked the top 3 in our study (Fig. $4 \mathrm{~b}$ ). The $\mathrm{MA}+\mathrm{CM}$ was recommended as the most effective intervention in improving the quality of life based on the 9 treatments from the Bayesian meta-analysis. Moreover, 8 interventions (MA + CM, AM, WA, SA+CM, MA, EA, Mox, CM) were significantly more effective than SA (Table 7)

\section{Reduction in $\lg E$}

Using STATA 15.0, we generated a network graph between the 9 therapies (Fig. 3c). According to the PSRF results (value close to 1, Additional file 1: Appendix S3 (a)) and P-values (Additional file 1: Appendix S3 (b)), we performed the Bayesian analysis via the consistency model, and the figure of ranking probability was generated (Fig. 4c). Mox, EA, and WA methods ranked the top 3 in the study (Fig. 4c). Moxibustion was recommended as the most effective intervention in changing the level of Ig $\mathrm{E}$ of the 9 treatments. As shown in Table 8, 7 interventions (Mox, EA, WA, MA+CM, FA+MA, FA, AM) were significantly more effective than CM, meanwhile, compared with CM, MA has little effect in reduction of Ig E. 

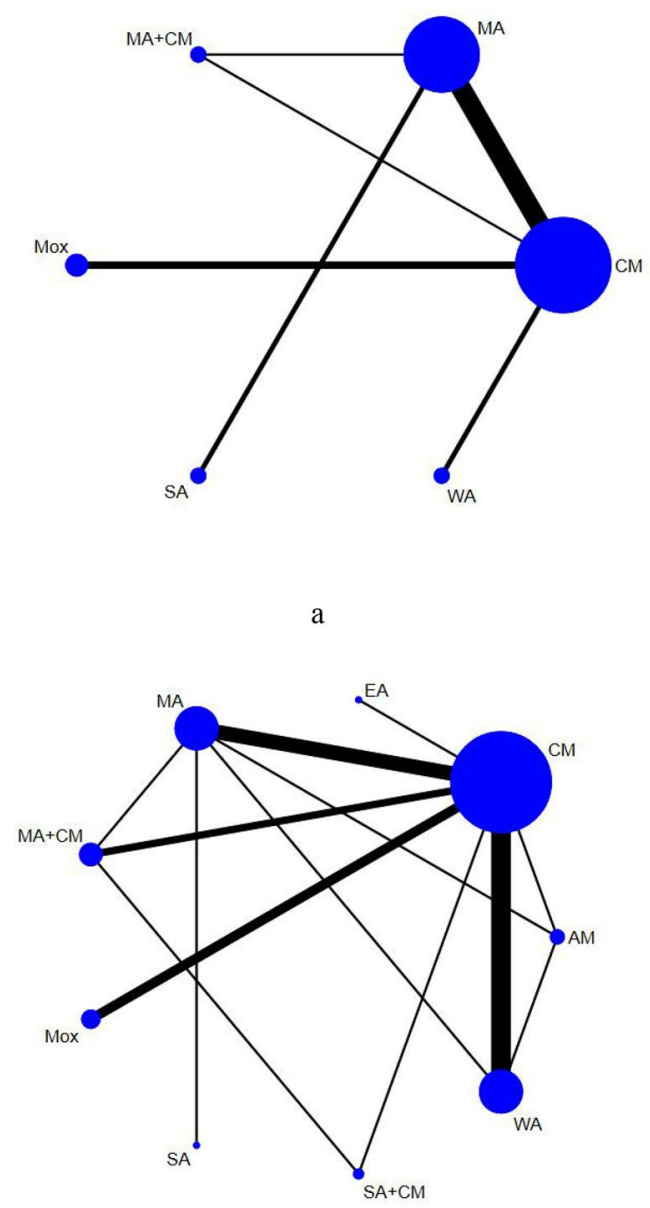

b

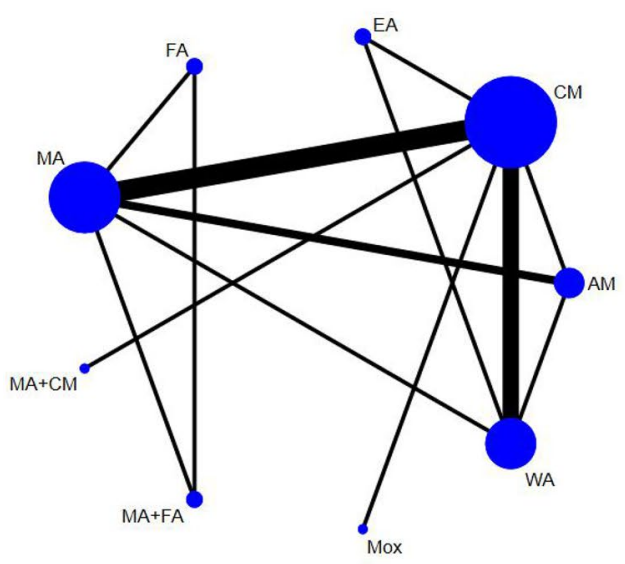

Fig. 3 a The network graph of different interventions of TNSS; $\mathbf{b}$ The network graph of different interventions of reduction in RQLQ; $\mathbf{c}$ The network graph of different interventions of the $\lg \mathrm{E}$

\section{Safety}

Notably, 16 RCTs [41, 44, 45, 50, 53, 55, 57, 60, 61, 64-66, $68,71,73,74]$, with 1,201 patients described information about safety. However, there were few adverse events associated with interventions (Table 9). The therapies included MA, WA, SA, CM. Acupuncture caused minor comfort, pain, headache, and skin trauma. Besides, CM caused and lethargy, headache, stomachache, and thirst. These AEs were acceptable, though no severe AEs occurred.

\section{Heterogeneity}

We conducted a sensitivity analysis using Revman to assess the stability and reliability of the joint meta-analysis results. Consequently, we found that our meta-analysis results were relatively stable. We appraised some factors, such as acupuncture methods, acupoints, duration of treatment among others to be different, which may cause high clinical heterogeneity.

\section{Publication bias}

The publication bias was evaluated by comparing the symmetry of the funnel plot. As shown in Figures in Fig. 5 for comparison-adjusted funnel graphs, the funnel graph illustrated that most of the RCTs were roughly symmetrically distributed on both sides of the midline. This demonstrated the decreased likelihood of small sample effects. However, there was no strong and powerful evidence of these small study effects across the outcomes.

\section{Discussion}

Numerous studies have described the efficacy and safety of acupuncture for treating AR, a dominant disease across the globe. Despite a diverse number of acupuncture methods currently being applied, these methods have not been standardized. Consequently, clinicians are forced to combine several acupuncture-based methods, which is time-consuming and associated with high financial costs as well as wastage of medical resources.

In this systematic review, upon comparing the efficacy of different acupuncture interventions for AR, we yielded reliable results $[77,78]$. For instance, Mox and MA + CM showed highly statistically significant differences in improving TNSS compared to CM when we conducted a pairwise meta-analysis. Moreover, the network Bayesian meta-analysis indicated Mox as the optimal therapy in reducing TNSS. When MA was integrated with CM, a higher reduction in the quality of life was realized compared with MA, CM, and SA+MA. MA and SA displayed significant differences, while $\mathrm{SA}+\mathrm{CM}$ was more effective than CM. Both AM and WA showed significant differences with CM. Moreover, the MA plus CM was 


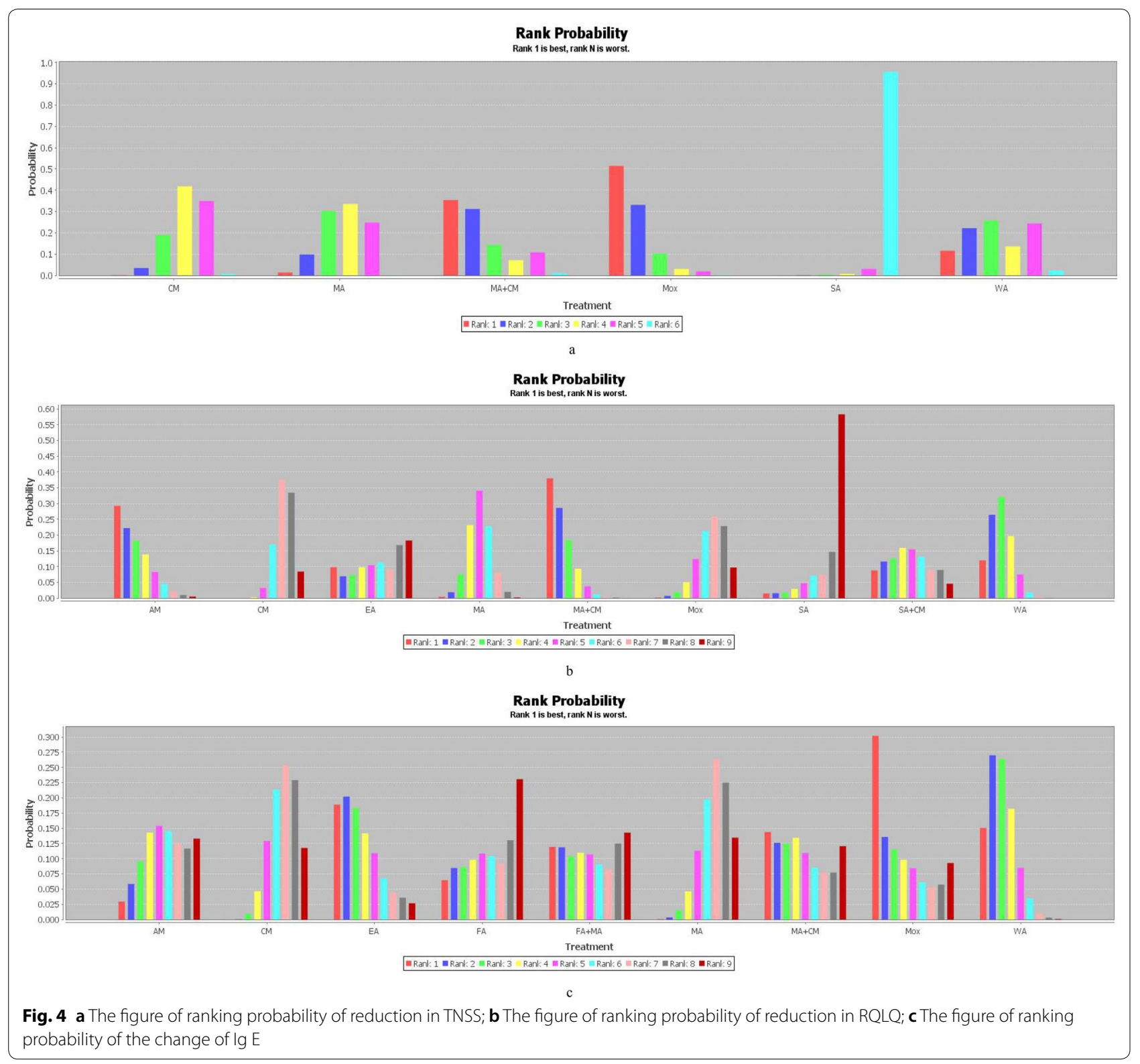

Table 6 The results of network meta-analysis of reduction of TNSS

\begin{tabular}{|c|c|c|c|c|c|}
\hline Mox & & & & & \\
\hline $0.80(-6.05,7.76)$ & $\mathrm{MA}+\mathrm{CM}$ & & & & \\
\hline $3.00(-1.37,7.49)$ & $2.21(-3.62,7.87)$ & MA & & & \\
\hline $3.31(0.45,7.26)$ & $2.55(-3.09,8.27)$ & $0.33(-2.01,2.68)$ & CM & & \\
\hline $2.42(0.45,7.27)$ & $1.63(-5.76,9.27)$ & $-0.62(-5.86,4.64)$ & $0.95(-3.85,5.69)$ & WA & \\
\hline $9.31(2.95,16.07)$ & $8.52(1.41,16.35)$ & $6.32(1.54,11.43)$ & $5.97(0.76,11.56)$ & $6.93(-0.12,14.16)$ & SA \\
\hline
\end{tabular}

Italic values indicate significant difference

implicated as the most effective therapy in the reduction of RQLQ. Regarding the IgE, we found significant differences between MA plus CM in pairwise meta-analysis.
Additionally, the Mox was indicated as the most effective treatment that changes the IgE content. Moreover, 16 RCTs $(41.03 \%)$ reported safety in adopting these 
Table 7 The results of network meta-analysis of quality of life (RQLQ)

\begin{tabular}{|c|c|c|c|c|c|c|c|c|}
\hline \\
\hline \multicolumn{9}{|l|}{$\begin{array}{l}M A+C M \\
3.62(-11.48, \\
19.07)\end{array}$} \\
\hline $\begin{array}{l}2.15(-17.89 \\
23.24)\end{array}$ & $\begin{array}{l}1.41(-15.90 \\
18.27)\end{array}$ & AM & & & & & & \\
\hline $\begin{array}{l}9.65(-10.86 \\
29.68)\end{array}$ & $\begin{array}{l}6.13(-16.20 \\
27.73)\end{array}$ & $\begin{array}{l}7.63(-19.18 \\
33.36)\end{array}$ & $\mathrm{SA}+\mathrm{CM}$ & & & & & \\
\hline $\begin{array}{l}11.07(-3.46, \\
26.04)\end{array}$ & $\begin{array}{l}7.54(-3.86 \\
19.16)\end{array}$ & $\begin{array}{c}9.09(-8.51 \\
26.08)\end{array}$ & $\begin{array}{l}1.46(-20.01 \\
23.30)\end{array}$ & MA & & & & \\
\hline $\begin{array}{l}14.75(-15.74 \\
44.58)\end{array}$ & $\begin{array}{l}11.15(-17.73 \\
39.24)\end{array}$ & $\begin{array}{l}12.42(-19.01 \\
44.06)\end{array}$ & $\begin{array}{l}4.96(-28.87 \\
39.14)\end{array}$ & $\begin{array}{l}3.45(-25.30, \\
32.29)\end{array}$ & $E A$ & & & \\
\hline $\begin{array}{c}17.36(0.51 \\
34.82)\end{array}$ & $\begin{array}{l}13.83(-0.15 \\
27.85)\end{array}$ & $\begin{array}{l}15.19(-5.28 \\
35.01)\end{array}$ & $\begin{array}{l}7.78(-15.34 \\
31.51)\end{array}$ & $\begin{array}{l}6.20(-8.19 \\
20.86)\end{array}$ & $\begin{array}{l}2.64(-26.75 \\
32.19)\end{array}$ & Mox & & \\
\hline $\begin{array}{l}18.80(6.31 \\
32.02)\end{array}$ & $\begin{array}{l}15.32(7.19 \\
23.62)\end{array}$ & $\begin{array}{l}16.71(0.24 \\
32.65)\end{array}$ & $\begin{array}{l}9.24(-10.79 \\
30.01)\end{array}$ & $\begin{array}{l}7.72(-1.14 \\
16.76)\end{array}$ & $\begin{array}{l}4.15(-22.50 \\
31.49)\end{array}$ & $\begin{array}{l}1.52(-9.92 \\
12.92)\end{array}$ & $C M$ & \\
\hline $\begin{array}{l}26.53(-2.62 \\
54.85)\end{array}$ & $\begin{array}{l}22.92(-4.16 \\
49.63)\end{array}$ & $\begin{array}{l}24.43(-6.30 \\
53.77)\end{array}$ & $\begin{array}{l}16.86(-16.10 \\
49.79)\end{array}$ & $\begin{array}{l}15.55(-9.59, \\
39.13)\end{array}$ & $\begin{array}{l}11.97(-25.96 \\
48.87)\end{array}$ & $\begin{array}{l}9.30(-20.18, \\
36.80)\end{array}$ & $\begin{array}{l}7.81(-19.31 \\
33.01)\end{array}$ & SA \\
\hline
\end{tabular}

Italic values indicate significant difference

$M A$ manual acupuncture, EA electroacupuncture, WA warm acupuncture, Mox moxibustion, $A M$ Acupuncture-Moxibustion, $S A$ sham acupuncture, $C M$ conventional medicine

Table 8 The results of network meta-analysis of $\lg E$

\begin{tabular}{|c|c|c|c|c|c|c|c|}
\hline \multicolumn{8}{|l|}{ WA } \\
\hline $\begin{array}{l}3.11(-92.21 \\
98.28)\end{array}$ & Mox & & & & & & \\
\hline $\begin{array}{l}3.61(-52.13 \\
62.15)\end{array}$ & $\begin{array}{l}0.51(-105.71 \\
106.01)\end{array}$ & EA & & & & & \\
\hline $\begin{array}{c}16.89(-66.06 \\
104.69)\end{array}$ & $\begin{array}{l}13.57(-101.21 \\
135.68)\end{array}$ & $\begin{array}{l}13.66(-81.95 \\
112.02)\end{array}$ & $\mathrm{MA}+\mathrm{CM}$ & & & & \\
\hline $\begin{array}{l}23.73(-65.51 \\
118.96)\end{array}$ & $\begin{array}{l}20.30(-101.69 \\
146.41)\end{array}$ & $\begin{array}{l}19.93(-83.46 \\
124.12)\end{array}$ & $\begin{array}{l}7.14(-114.30 \\
125.63)\end{array}$ & $\mathrm{FA}+\mathrm{MA}$ & & & \\
\hline $\begin{array}{l}30.35(-26.32 \\
92.74)\end{array}$ & $\begin{array}{l}27.35(-76.58 \\
133.25)\end{array}$ & $\begin{array}{l}26.19(-49.60 \\
106.14)\end{array}$ & $\begin{array}{l}\text { 12.78 (-81.05 } \\
107.77)\end{array}$ & $\begin{array}{l}6.63(-91.93 \\
106.78)\end{array}$ & AM & & \\
\hline $\begin{array}{l}33.48(-55.68 \\
127.08)\end{array}$ & $\begin{array}{l}30.54(-92.75 \\
155.05)\end{array}$ & $\begin{array}{l}30.32(-73.22 \\
134.01)\end{array}$ & $\begin{array}{l}16.50(-100.39 \\
133.05)\end{array}$ & $\begin{array}{l}10.19(-69.46 \\
90.97)\end{array}$ & $\begin{array}{l}3.98(-95.17 \\
99.06)\end{array}$ & FA & \\
\hline $40.43(7.26,76.07)$ & $\begin{array}{l}37.74(-50.49 \\
126.01)\end{array}$ & $\begin{array}{l}36.78(-22.30 \\
93.11)\end{array}$ & $\begin{array}{l}23.42(-55.38 \\
100.04)\end{array}$ & $\begin{array}{l}16.94(-72.87 \\
102.00)\end{array}$ & $\begin{array}{l}10.30(-46.19 \\
62.39)\end{array}$ & $\begin{array}{l}6.74(-79.89 \\
91.96)\end{array}$ & $\mathrm{CM}$ \\
\hline $\begin{array}{l}41.25(-1.79 \\
87.41)\end{array}$ & $\begin{array}{l}38.36(-55.33 \\
131.83)\end{array}$ & $\begin{array}{l}37.80(-28.52 \\
101.34)\end{array}$ & $\begin{array}{l}24.34(-61.11 \\
108.24)\end{array}$ & $\begin{array}{l}17.61(-64.84 \\
97.82)\end{array}$ & $\begin{array}{l}11.12(-45.79 \\
64.63)\end{array}$ & $\begin{array}{l}8.17(-74.48 \\
88.90)\end{array}$ & $\begin{array}{l}0.82 \\
(-30.25 \\
32.96)\end{array}$ \\
\hline
\end{tabular}

Italic values indicate significant difference

interventions. However, only 7 RCTs about acupuncture were associated with adverse events (minor comfort, pain, skin trauma, etc.) which were acceptable, though no severe adverse events occurred.

This systematic review has some limitations. Firstly, we purposed to reduce bias, however, it was unclear whether other potential studies were included. Most of the included RCTs were investigated in China, and the search language was limited to Chinese/English. Thus, this could potentially cause bias. Secondly, we, in most cases got 1 or 2 small trials to compare the effect of acupuncture methods, this could lead to insufficient statistical efficiency. Thirdly, some acupuncture studies had a challenge in determining the risk of bias. Therefore, researchers should be attempted to minimize bias. Additionally, acupuncture may result in numerous factors, such as the selection of acupoint, the treatment duration, among others. Meanwhile, the CM has differences in type, dosage, dosage forms which might cause heterogeneity. Moreover, most of the included trials [38, 47, 48, $51,55,60,62]$ had a short treatment duration and lacked 
Table 9 Adverse events in included studies

\begin{tabular}{|c|c|c|c|}
\hline Interventions & Sample size & Study & Results \\
\hline \multirow[t]{3}{*}{ MA } & 18 & Lan 2010 [73] & A: 1 case of inconsequential bleeding \\
\hline & 42 & Xue 2008 [74] & A: 11 cases of minor comfort, 1 case of headache, 1 case of dizziness \\
\hline & 32 & Yu 2015 [64] & A: 2 cases with pain \\
\hline \multirow[t]{4}{*}{ WA } & 105 & Sun 2020 [41] & A: 1 cases of dizziness, 2 cases fear acupuncture and moxibustion \\
\hline & 30 & Zhang 2019 [44] & A: 1 case with dizziness, 1 case with blister \\
\hline & 49 & Gao 2019 [45] & A: 3 cases of skin trauma, 2 cases of itchiness \\
\hline & 27 & Wang 2013 [71] & A: 1case of inconsequential bleeding \\
\hline \multirow[t]{2}{*}{ SA } & 18 & Lan 2010 [73] & B: 1 case of dizziness \\
\hline & 38 & Xue 2008 [74] & B: 8 cases of minor comfortable, 2 cases of headache \\
\hline \multirow[t]{2}{*}{ CM } & 105 & Sun 2020 [41] & B: 1 case of headache, 1 case of stomachache, 1 case of thirst \\
\hline & 32 & Yu 2015 [64] & B: 2 cases with drowsiness \\
\hline
\end{tabular}

$M A$ manual acupuncture, WA warm acupuncture, $S A$ sham acupuncture, $C M$ conventional medicine

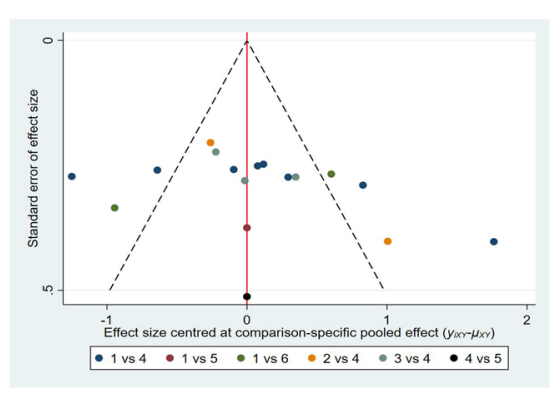

Note. MA: 1; WA: 2; Mox: 3; CM: 4; MA+CM: 5; SA: 6

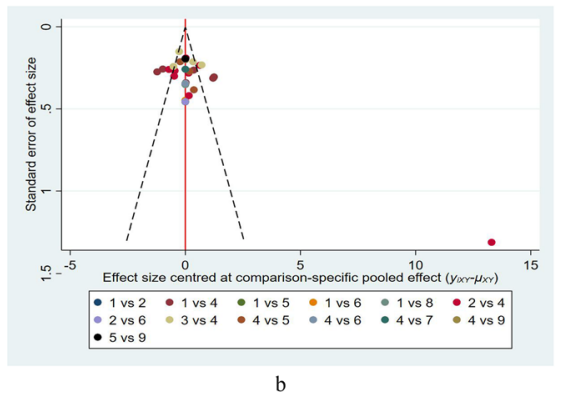

Note. MA: 1; WA: 2; Mox: 3; CM: 4; MA+CM: 5; AM: 6; EA: 7; SA: 8; SA+CM:9

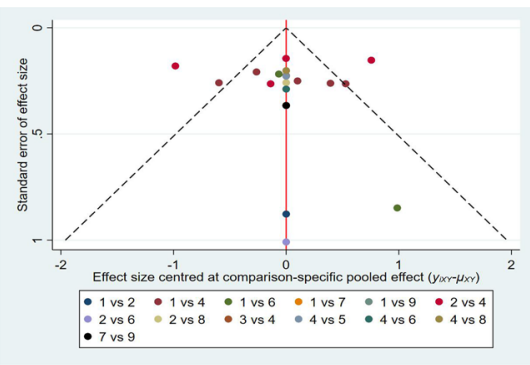

Note. MA: 1; WA: 2; Mox: 3; CM: 4; MA+CM: 5; AM: 6; FA: 7; EA: 8; MA+FA: 9

Fig. 5 a Funnel plot for the network meta-analysis of reduction in TNSS; b Funnel plot for the network meta-analysis of reduction in RQLQ; c Funnel plot for the network meta-analysis of change of lg $E$ follow-up, which is still insufficient to illustrate the longterm effect of acupuncture.

Several previous trials demonstrated the clinical efficacy and safety of acupuncture for AR. However, numerous outcomes of the trials were dependent on the subjective records and feelings of these participants. Besides, because of the various interventions of acupuncture, inconsistencies occur in the selection of acupoint, duration, and frequency of treatment, among others. These factors may cause heterogeneity. Therefore, future research should be geared towards standardizing and generalizing acupuncture methods, acupoint, duration, and frequency of AR treatment. Moreover, since the methodological quality in our study was low to moderate, a well-designed RCTs should be implemented with the Consolidated Standards of Reporting Trials (CONSORT), Standards for Reporting Interventions in Clinical Trials of Acupuncture (STRICTA), Standards for Reporting Interventions in Clinical Trials of Moxibustion (STRICTOM) and Cochrane Handbook for Systematic Reviews of Interventions [79-81] to command the quality of future studies [82, 83]. Moreover, we suggest that protocols should be registered. The methodological quality of the included trials was not satisfactory, thus more precise and accuracy designed, generated, and published RCTs are warranted. Of note, we also wish future scholars to select outcome measures based on international consensus.

\section{Conclusion}

This work identifies acupuncture as one of several effective therapies for AR. MA+CM/Mox may effectively improve AR symptoms and quality of life as demonstrated in pairwise and Bayesian network meta-analyses. 
Meanwhile, Mox was regarded as the most effective therapy that changes the IgE content from recently evidences. However, insufficient clinical evidence is presently available to guide on the selection of the acupoints, duration of treatment among others. Meanwhile, the overall quality of these included RCTs were mainly ranked as moderate. Therefore, lots of high-quality RCTs are required to validate the above-presented findings.

\section{Supplementary information}

Supplementary information accompanies this paper at https://doi. org/10.1186/s13020-020-00389-9.

Additional file 1: Appendix S1. The PSRF value of reduction in TNSS. Appendix S2. (a) The PSRF value of reduction in RQLQ. (b) Node-splitting test result of reduction in RQLQ. Appendix S3. (a) The PSRF value of reduction in $\lg \mathrm{E}$. (b) Node-splitting test result of reduction in $\lg \mathrm{E}$.

\section{Abbreviations}

AR: Allergic rhinitis; IgE: Immunoglobulin E; WOS: Web of science; CNKI: China National Knowledge Infrastructure; VIP: China Science and Technology Journal Database; CBM: Chinese Biomedical Literature Database; WHO ICTRP: World Health Organization International Clinical Trials Registry Platform; ChiCTR : Chinese Clinical Trial Register; AMED: Allied and Complementary Medicine Database; ADDIS: Aggregate Data Drug Information System; NMA: Network meta-analysis; ICC: Intra-class correlation coefficient; TNSS: Total nasal symptom score; RQLQ: Rhinoconjunctivitis quality of life questionnaire; MA: Manual acupuncture; SA: Sham acupuncture; CM: Conventional medicine; RCTs: Randomized controlled trials; Mox: Moxibustion; EA: Electronic acupuncture; WA: Warm acupuncture; AM: Acupuncture-moxibustion; FA: Fire acupuncture; PRISMA: Preferred Reporting Items for Systematic Reviews and Meta-analyses; AE: Adverse event; Cl: Confidence interval; SMD: Standard mean difference; MCMC: Markov Chain Monte Carlo; PSRF: Potential scale reduced factor; Revman: Review manager; WF: Wanfang Database.

\section{Acknowledgements}

\section{Not applicable.}

\section{Authors' contributions}

$Z Y, L Z$, and FL conceived this study. ZY developed and implemented the systematic review, under the supervision of LZ. ZY provided the statistical analysis plan for the study and performed data analysis. GG and GX performed study search, screening, and extraction of data, whereas $L Z$ reviewed the work. ZY wrote the first draft of the current review, with $L Z$ and $F L$ provided input to the final draft. All authors read and approved the final manuscript.

\section{Funding}

This work was financially supported by the National key research and development program of China (No. 2019YFC1709700), the National Natural Science Foundation of China (Nos. 81590951, 81722050, and 81973961) and the Project of Science and Technology Department of Sichuan Province (Nos. 20ZDYF1199 and 2019YFS0081).

\section{Ethics approval and consent to participate}

Findings from the study will be disseminated through a peer-reviewed article.

\section{Consent for publication}

Not applicable.

\section{Competing interests}

The authors declare that they have no competing interests.

Received: 31 July 2020 Accepted: 26 September 2020 Published online: 12 October 2020

\section{References}

1. Greiner AN, Hellings PW, Rotiroti G, et al. Allergic rhinitis. Lancet. 2011;378(9809):2112-22.

2. Bousquet J, Khaltaev N, Cruz AA, et al. Allergic rhinitis and its impact on asthma (ARIA) 2008 update (in collaboration with the World Health Organization, GA(2)LEN and AllerGen). Allergy. 2008;63(Suppl 86):8-160.

3. Bousquet PJ, Demoly P, Devillier P, Mesbah K, Bousquet J. Impact of allergic rhinitis symptoms on quality of life in primary care. Int Arch Allergy Immunol. 2013:160(4):393-400.

4. Kakli HA, Riley TD. Allergic rhinitis. Prim Care. 2016;43(3):465-75.

5. Bernstein DI, Schwartz G, Bernstein JA. Allergic Rhinitis: Mechanisms and Treatment[J]. Immunol Allergy Clin North Am. 2016;36(2):261-78.

6. Meltzer EO, Blaiss MS, Naclerio RM, et al. Burden of allergic rhinitis: allergies in America, Latin America, and Asia-Pacific adult surveys. Allergy Asthma Proc. 2012;33:S113-S14141.

7. Greiner AN, Hellings PW, Rotiroti G, Scadding GK. Allergic rhinitis. Lancet. 2011;378(9809):2112-22.

8. Zhang Y, Zhang L. Prevalence of allergic rhinitis in china. Allergy Asthma Immunol Res. 2014;6(2):105-13.

9. Wang XD, Zheng M, Lou HF, et al. An increased prevalence of selfreported allergic rhinitis in major Chinese cities from 2005 to 2011. Allergy. 2016;71(8):1170-80.

10. Zhang $Y$, Zhang L. Increasing prevalence of allergic rhinitis in China. Allergy Asthma Immunol Res. 2019;11(2):156-69.

11. Meltzer EO. Allergic rhinitis: burden of illness, quality of life, comorbidities, and control. Immunol Allergy Clin North Am. 2016;36:235-48.

12. Crystal-Peters J, Crown WH, Goetzel RZ, Schutt DC. The cost of productivity losses associated with allergic rhinitis. Am J Manag Care. 2000;6:373-8.

13. Seidman MD, Gurgel RK, Lin SY, et al. Clinical practice guideline: allergic rhinitis. Otolaryngol Head Neck Surg. 2015;152(2):197-206.

14. Blaiss MS. Allergic rhinitis: direct and indirect costs. Allergy Asthma Proc. 2010;31:375-80.

15. Schofield ML. Asthma pharmacotherapy. Otolaryngol Clin North Am. 2014;47:55-64.

16. Mi J, Chen $X$, Lin $X$, et al. Treatment of persistent allergic rhinitis via acupuncture at the sphenopalatine acupoint: a randomized controlled trial. Trials. 2018;19(1):28.

17. Chen Y, Jin X, Yu M, et al. Zhongguo Zhen Jiu. 2015;35(4):339-343

18. May JR, Dolen WK. Management of allergic rhinitis: a review for the community pharmacist. Clin Ther. 2017;39:2410-9.

19. Cheng L, Chen J, Fu Q, et al. Chinese society of allergy guidelines for diagnosis and treatment of allergic rhinitis. Allergy Asthma Immunol Res. 2018;10(4):30.

20. Feng S, Han M, Fan Y, et al. Acupuncture for the treatment of allergic rhinitis: a systematic review and meta-analysis. Am J Rhinol Allergy. 2015;29(1):57-62.

21. Fu Q, Zhang L, Liu Y, et al. Effectiveness of acupuncturing at the sphenopalatine ganglion acupoint alone for treatment of allergic rhinitis: a systematic review and meta-analysis. Evid Based Complement Alternat Med. 2019;2019:6478102.

22. Chen $Y D$, Jin $X Q$, Yu MH, Fang $Y$, Huang LQ. Acupuncture for moderate to severe allergic rhinitis: a non-randomized controlled trial. Chin J Integr Med. 2016;22(7):518-24.

23. Adam D, Grabenhenrich L, Ortiz M, Binting S, Reinhold T, Brinkhaus B. Impact of acupuncture on antihistamine use in patients suffering seasonal allergic rhinitis: secondary analysis of results from a randomised controlled trial. Acupunct Med. 2018;36(3):139-45.

24. Xue CC, Zhang AL, Zhang CS, DaCosta C, Story DF, Thien FC. Acupuncture for seasonal allergic rhinitis: a randomized controlled trial. Ann Allergy Asthma Immunol. 2015;115(4):317-324.e1.

25. Ding SS, Hong SH, Wang C, Guo Y, Wang ZK, Xu Y. Acupuncture modulates the neuro-endocrine-immune network. QJM. 2013;107(5):341-5.

26. Chen B, Ming-Yue LI, Dind SS, et al. Research progress on regulations on nerve-endocrine-immune network by acupuncture. World J Acupunct Moxibustion. 2014;24(4):49-58.

27. Taw MB, Reddy WD, Omole FS, Seidman MD. Acupuncture and allergic rhinitis. Curr Opin Otolaryngol Head Neck Surg. 2015;23(3):216-20.

28. Ipci K, Oktemer T, Muluk NB, et al. Alternative products to treat allergic rhinitis and alternative routes for allergy immunotherapy. Am J Rhinol Allergy. 2016;30(5):8-10. 
29. Chen S, Qu SH, Zhang Y, et al. Impact of acupuncture for allergic rhinitis on the activity of the hypothalamus-pituitary-adrenal axis: study protocol for a randomized controlled trial. Trials. 2019;20(1):372.

30. Liang Y, Lenon GB, Yang AWH. Acupressure for respiratory allergic diseases: a systematic review of randomised controlled trials. Acupunct Med. 2017;35(6):413-20.

31. Wen CY, Liu YF, Zhou L, Zhang HX, Tu SH. A systematic and narrative review of acupuncture point application therapies in the treatment of allergic rhinitis and asthma during dog days. Evid Based Complement Alternat Med. 2015;2015:846851.

32. Li XR, Zhang QX, Liu M, et al. Catgut implantation at acupoints for allergic rhinitis: a systematic review. Chin J Integr Med. 2014;20(3):235-40.

33. Zhang $C S$, Yang AW, Zhang AL, et al. Ear-acupressure for allergic rhinitis: a systematic review. Clin Otolaryngol. 2010;35(1):6-12.

34. Hutton B, Salanti G, Caldwell DM, Chaimani A, Schmid CH, Cameron C, et al. The PRISMA extension statement for reporting of systematic reviews incorporating network meta-analyses of health care interventions: checklist and explanations. Ann Intern Med. 2015;162(11):777-84.

35. Higgins JP, Altman DG, Gøtzsche PC, et al. The Cochrane Collaboration's tool for assessing risk of bias in randomised trials. BMJ. 2011;343:d5928.

36. Hua Z, Zhai FT, Tian J, et al. Effectiveness and safety of oral Chinese patent medicines as adjuvant treatment for unstable angina pectoris on the national essential drugs list of China: a protocol for a systematic review and network meta-analysis. BMJ Open. 2019;9(9):e026136.

37. Cipriani A, Higgins JPT, Geddes JR, et al. Conceptual and technical challenges in network meta-analysis. Ann Intern Med. 2013;159:130-7.

38. Hou F, Leng H, Wu JA, et al. Clinical study on the treatment of allergic rhinitis by acupuncture of sphenopalatine ganglion. J Liaoning Univ Tradit Chin Med. 2020;22(04):125-8.

39. Wu Z. Evaluation of the effect of acupuncture of sphenopalatine ganglion on allergic rhinitis. Chin Health Care. 2020;38(6):186-7.

40. Zhang L, Yang JH, Fu J. Clinical study on the treatment of allergic rhinitis with heavy - dose moxibustion. Hebei J TCM. 2020;42(1):101-5.

41. Sun DP, Ma XM, Jiang MX, et al. Therapeutic analysis of Da Aiduan warm needle moxibustion on 100 cases of allergic rhinitis with cold of deficiency pattern in Lung Qi. J Nanjing Univ Tradit Chin Med. 2020;36(1):19-23.

42. Song WJ, Feng X, Lai RM, et al. Observation on curative effect of acupuncture sphenopalatine ganglion point on perennial allergic rhinitis. China Med Pharm. 2020;10(5):36-9.

43. Wang SY, Zhang J, Du R, et al. Clinical observations on heat-sensitive point moxibustion for perennial allergic rhinitis. Shanghai J Acu-mox. 2020;39(06):734-8

44. Zhang HM. Clinical Study of Needle warming moxibustion in the Treatment of Allergic Rhinitis of Lung-Spleen Deficiency and Cold Syndrome [Master]. Chengdu University of Traditional Chinese Medicine; 2019.

45. Gao MY. Comparative study on clinical efficacy between acupuncture and moxibustion and loratadine in alleviating nasal symtoms for persistent allergic rhinitis [Master]. China Academy of Chinese Medical Sciences, 2019.

46. Liao JJ. Effect of Fuyang Tongqiao acupuncture on quality of life in patients with allergic rhinitis. Chin J Health Nutr. 2019;29(7):331.

47. Li Y, Wang DY, Han KC, et al. Clinical study of intranasal acupuncture treating allergic rhinitis. J Clin Acu-Mox. 2019;35(5):25-9.

48. Wang AP, Sun HB, Leng $H$, et al. Clinical Study of Warm Needling plus Desloratadine Citrate Disodium Tablets for Allergic Rhinitis. Shanghai J Acu-mox. 2019;38(9):1022-6.

49. Lu YL. Observation on the clinical effect of abdominal acupuncture regulating Shentongqiao method on moderate and severe allergic rhinitis [Master]. Beijing University of Chinese Medicine, 2018.

50. Zhao QY. Effect of abdominal acupuncture on nasal symptoms and quality of life in patients with moderate to severe allergic rhinitis [Master]. Beijing University of Chinese Medicine, 2018.

51. Yuan SX. Analysis of quality of life and observation of emotional condition in patients with the type of deficiency-cold allergic rhinitis treated by Warm acupuncture and moxibustion [Master]. Chengdu University of Traditional Chinese Medicine, 2018.

52. Fang YL. Therapeutic effect of treatment allergic rhinitis with acupuncture bilateral sphenopalatine ganglion. J Jiangxi Univ TCM. 2018;30(02):56-8.
53. Wen L. The effect of Peitushengjin warm acupuncture on the quality of life of patients with allergic rhinitis with spleen deficiency [Master]. Fujian University of Traditional Chinese Medicine, 2018.

54. Li YN. Moxibustion for the treatment of allergic rhinitis and its effect on quality of life. Mod I Integr Trad Chin West Med. 2018;27(27):3012-4.

55. Li YH, Huang YS. Clinical analysis of warm needling on the expression of serum specific lgE for patients with persistent allergic rhinitis of Lung-Qi deficiency and cold type. J Sichuan of TCM. 2018;36(4):174-6.

56. Li KL, Li WY, Chen JX, et al. Clinical observation on the treatment of severe perennial allergic rhinitis with acupuncture on sphenopalatine ganglion. Int J Trad Chin Med. 2018;40(4):309-13.

57. Zheng WB. Observation on the effect of Fuyang Tongqiao acupuncture on improving the quality of life in patients with allergic rhinitis [Master]. Beijing University of Chinese Medicine, 2017.

58. Cao SH, Mai YH, Pan RY. Observation of therapeutic effect of heatsensitive moxibustion combined with acupuncture on allergic rhinitis. J Practical TCM. 2017;33(6):718.

59. Li SN. Observation on the therapeutic effect of acupuncture and moxibustion Fuyang Guben method on persistent allergic rhinitis [Master]. Beijing University of Chinese Medicine, 2016.

60. Liu Y. Clinical study on warm acupuncture for treatment of persistent allergic rhinitis with deficiency of Lung Qi [Doctor]. Chengdu University of Traditional Chinese Medicine, 2016.

61. Li S. Observation of clinical curative effect of mild moxibustion on persistent allergic rhinitis [Master]. Beijing University of Chinese Medicine, 2016

62. Jin YJ. The Clinical observation of moxa cone moxibustion on lung's shu and mu acupoints for the treatment of allergic rhinitis of lung deficiency related cold type [Master]. Guangzhou University of Chinese Medicine, 2016.

63. Chen TT, Liu FH. Therapeutic observation of acupuncture at phenomaxillary ganglia for allergic rhinitis. Shanghai J Acu-mox. 2016;35(7):850-2.

64. Yu XH. Observation on clinical effect of acupuncture on sphenopalatine ganglion for allergic rhinitis [Master]. Beijing University of Chinese Medicine, 2015.

65. Chen C, Wang J, Bai P, et al. Moderate and severe persistent allergic rhinitis treated with acupuncture: a randomized controlled trial. Chin J Acu-Mox. 2015;35(12):1209-13.

66. He JZ. Controlled Clinical study of allergic rhinitis treated with transcutaneous electrical nerve stimulation on acupoints of nasal three-needle [Master]. Guangzhou University of Chinese Medicine; 2014.

67. Huang HH. Clinical study fire acupuncture on allergic rhinitis [Doctor]. Nanjing University of Chinese Medicine; 2014.

68. Si HF. The observation on clinical effectiveness of warm acupuncture in the treatment of allergic rhinitis [Master]. China Academy of Chinese Medical Sciences; 2014.

69. Zhang YC. Observation on clinical effect of acupuncture on allergic rhinitis [Master]. Beijing University of Chinese Medicine; 2013.

70. Shi ZH. Observation on clinical curative effect of "Tiaoshen acupuncture method" in treating allergic rhinitis [Doctor]. Beijing University of Chinese Medicine; 2013

71. Wang $P$, Luo H, Sun JQ, et al. A clinical observation on regulating spirit acupuncture for 27 cases of moderate to severe allergic rhinitis. J Trad Chin Med. 2013:54(24):2117-200.

72. Benno B, Miriam O, Claudia M, et al. Acupuncture in Patients With Seasonal Allergic Rhinitis A Randomized Trial. Ann Intern Med 2013;158(4):225-34. 36

73. Lan SY. Randomized controlled study on acupuncture treatment of persistent allergic rhinitis [Master]. Beijing University of Chinese Medicine; 2010.

74. Xue C, An XD, Cheung TP, et, al. Acupuncture for persistent allergic rhinitis: a randomised, sham-controlled trial; 2008.

75. Li YM, Lai XS, Zhuang LX, et, al. Observation on therapeutic effect of electroacupuncture on sphenopalatine ganglion in treating 50 cases of perennial allergic rhinitis. J New Chin Med. 2007;39(3).

76. Rao YQ, Han NY. Therapeutic effect of acupuncture on allergic rhinitis and its effects on immunologic function. Chin J Acu-Mox. 2006;26(8):557-60.

77. Caldwell DM, Ades AE, Higgins JPT. Simultaneous comparison of multiple treatments: combining direct and indirect evidence. BMJ. 2005;331:897-900.

78. Salanti G. Indirect and mixed-treatment comparison, network, or multiple-treatments meta-analysis: many names, many benefits, many 
concerns for the next generation evidence synthesis tool. Res Synth Methods. 2012;3:80-97.

79. Zhang YJ, Cao HJ, Li XL, et al. Cupping therapy versus acupuncture for pain-related conditions: a systematic review of randomized controlled trials and trial sequential analysis. Chin Med. 2017;12:21.

80. Svenkerud S, MacPherson H. The impact of STRICTA and CONSORT on reporting of randomised control trials of acupuncture: a systematic methodological evaluation. Acupunct Med. 2018;36(6):349-57.

81. Cheng CW, Fu SF, Zhou QH, et al. Extending the CONSORT Statement to moxibustion. J Integr Med. 2013;11(1):54-63.

82. Shi J, Hu H, Harnett J, et al. An evaluation of randomized controlled trials on nutraceuticals containing traditional Chinese medicines for diabetes management: a systematic review. Chin Med. 2019;14:54
83. Qian $G$, Zeng J, Lu L, et al. Evaluation of reporting quality in randomised controlled trials of acupuncture for acute herpes zoster by the CONSORT statement and STRICTA guidelines. Evid Based Complement Alternat Med. 2020;2020:4308380.

\section{Publisher's Note}

Springer Nature remains neutral with regard to jurisdictional claims in published maps and institutional affiliations.
Ready to submit your research? Choose BMC and benefit from:

- fast, convenient online submission

- thorough peer review by experienced researchers in your field

- rapid publication on acceptance

- support for research data, including large and complex data types

- gold Open Access which fosters wider collaboration and increased citations

- maximum visibility for your research: over $100 \mathrm{M}$ website views per year

At BMC, research is always in progress.

Learn more biomedcentral.com/submissions 